\title{
Simultaneous Vibration and Noise Reduction in Rotorcraft - Practical Implementation Issues
}

\author{
Li Liu*, Peretz P. Friedmann ${ }^{\dagger}$ and Dan Patt \\ Department of Aerospace Engineering, The University of Michigan, Ann Arbor, MI, 48109, USA
}

\begin{abstract}
A study of helicopter vibration and blade-vortex interaction (BVI) noise reduction is conducted using the actively-controlled trailing edge flap (ACF) approach implemented in single and dual flap configurations. The effectiveness of a passive approach based on varying the sweep on the tip of the rotor to modify noise and vibration characteristics of the rotor is also considered. The study is based on a comprehensive rotorcraft aeroelastic/aeroacoustic simulation code that was validated against the experimental data obtained in the Higherharmonic-control Aeroacoustic Rotor Test (HART) program. The effectiveness of the ACF system for vibration and noise reduction is explored on two different helicopter configurations, one resembling a four-bladed MBB BO-105 hingeless rotor and the other similar to a five-bladed MD-900 bearingless rotor. Issues associated with the practical implementation of the ACF approach are emphasized and examined such as: the effects of practical flap saturation limits, constant and $1 /$ rev control inputs and flap overhang. The simulation results demonstrate the capability of the ACF system for effective vibration and BVI noise reduction.
\end{abstract}

\section{Nomenclature}

$\begin{array}{ll}c & \text { Blade chord } \\ C_{T} & \text { Rotor thrust coefficient } \\ C_{W} & \text { Helicopter weight coefficient } \\ \mathbf{D} & \text { Matrix defined to be } \mathbf{T}^{\mathrm{T}} \mathbf{Q T}+\mathbf{R} \\ F_{H X}, F_{H Y}, & \\ \quad F_{H Z} & \text { Nondimensional } N_{b} / \mathrm{rev} \text { hub shears } \\ J\left(\mathbf{z}_{k}, \mathbf{u}_{k}\right) & \text { Objective function } \\ k & \text { Control update index } \\ L_{b} & \text { Blade length } \\ L_{c} & \text { Control surface length } \\ M_{H X}, M_{H Y}, & \\ M_{H Z} & \text { Nondimensional } N_{b} / \text { rev hub moments } \\ M_{\text {ref }} & \text { Reference mass } \\ N & \text { Number of flap deflection input harmonic } \\ N_{b} & \text { Number of rotor blades } \\ N_{H 06}, \cdots, N_{H 17} & \text { Noise levels of the } 6^{\text {th }}-17^{\text {th }} \text { harmonics of blade passage frequency } \\ \mathbf{Q} & \text { Weighting matrix for objectives to be reduced } \\ \mathbf{R} & \text { Weighting matrix on control input } \\ R & \text { Rotor radius } \\ \mathbf{T} & \text { Sensitivity, transfer matrix between control inputs and objective function } \\ \mathbf{u}_{k} & \text { Control input vector, } k^{\text {th }} \text { control step } \\ \mathbf{u}_{k, \text { opt }} & \text { Optimum value of control input vector }\end{array}$

*Ph.D. Candidate, Student Member aIAA.

$\dagger$ François-Xavier Bagnoud Professor, Fellow AIAA, AHS

$\ddagger$ Postdoctoral Researcher, Member AIAA. 


$\begin{array}{ll}x_{c} & \text { Spanwise location of center of control surface } \\ \mathbf{z}_{k} & \text { Objective vector, } k^{\text {th }} \text { control step } \\ \alpha & \text { Rotor disk angle of attack } \\ \alpha^{\prime} & \text { Effective rotor tip-path plane angle, corrected for wind tunnel effects } \\ \alpha_{R} & \text { Relaxation coefficient for control algorithm } \\ \beta_{p} & \text { Blade precone angle } \\ \delta & \text { Flap deflection angle } \\ \delta_{N c}, \delta_{N s} & \text { N/rev cosine and sine amplitude of } \delta, \text { respectively } \\ \delta_{f} & \text { Prescribed flap deflection angle } \\ \Lambda_{a} & \text { Tip inclination angle from the horizontal, positive upward(dihedral) } \\ \Lambda_{s} & \text { Tip sweep angle, positive backward } \\ \mu & \text { Helicopter advance ratio } \\ \Omega & \text { Rotor angular speed } \\ \omega_{F}, \omega_{L}, \omega_{T} & \text { Rotating flap, lead-lag and torsional frequencies } \\ \psi & \text { Rotor azimuth angle } \\ \sigma & \text { Rotor solidity } \\ \theta_{\mathrm{FP}} & \text { Flight path angle } \\ \theta_{\mathrm{tw}} & \text { Built-in twist angle }\end{array}$

\section{Introduction}

T imitations on noise and vibration levels in rotorcraft have increased in stringency, motivated by the Udesire for smooth ride in helicopters combined with the goal of improving the community acceptance of rotorcraft in densely populated areas. All new helicopters must meet demanding FAA flyover noise level tests, and desirable vibration levels stipulated by what is known as the intrusion index, that tends to limit vertical acceleration levels below $0.05 \mathrm{~g}$. Furthermore, active noise and vibration reduction systems must be implemented without undue performance penalties, so as to reap the largest potential benefit on the fairly sizeable cost associated with installing such active control systems in rotorcraft. While some of these statements apply primarily to civilian operations, similar demands for military operation are driven by pilot fatigue, maintenance costs, weapon system accuracy and the reduction of the noise footprint for stealth purposes.

These requirements have motivated a significant body of research on active vibration reduction ${ }^{1,2}$ as well as noise reduction. ${ }^{3}$ Noise and vibration generation are intrinsically linked as they are fundamentally driven by the same phenomena namely, unsteady aerodynamic loading and blade motion. Despite these common origins, however, the simultaneous noise and vibration generation/reduction problem is not well understood, and its implementation on different rotor configurations has not been explored in previous studies. This paper addresses this problem primarily for a flight condition where blade vortex interaction (BVI) is important. Blade-vortex interaction occurs when a blade encounters the concentrated vorticity trailed by another blade. This phenomenon is usually associated with low speed $(\mu \approx 0.15)$ descending flight, when the trailed rotor wake is closely spaced and likely to lie largely in the plane of the rotor. The interaction generates large unsteady pressure fluctuations on the blade that produce high levels of vibration and noise. The vibration levels often exceed those present at higher advance ratios $(\mu \approx 0.30)$ in level flight. The noise generated by BVI has several distinctive characteristics. It tends to have a strong directivity pattern focused mostly forward and under the rotor plane, ${ }^{3}$ making it particularly apparent to an observer on the ground ahead of a descending helicopter. The noise is also periodic, with a frequency content typically defined to be the 6th through 40th harmonics of blade passage frequency, in the mid-frequency range, generally considered to be most annoying to human hearing. Figure 1 depicts a typical blade-vortex interaction and defines the properties of miss-distance and interaction angle.

There are a number of factors governing BVI events:

1. The advance ratio, rate of descent, and rotor angular speed all affect the geometry of the trailed wake, and thus the strength and type of BVI. Noise from BVI is most severe when the wake is trailed directly into the plane of the rotor and oncoming blades.

2. The magnitude of pressure fluctuations on the rotor blade have a strong effect on the magnitude of BVI 


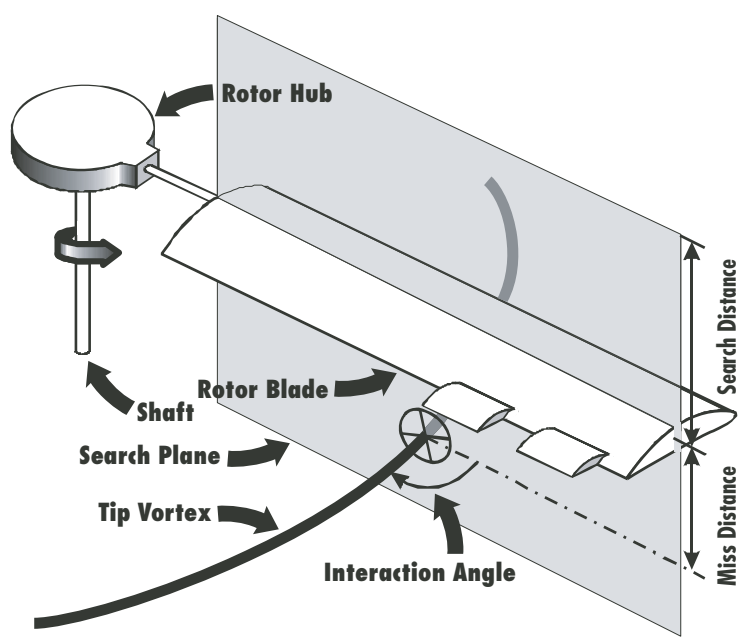

Figure 1. BVI intersection showing miss distance and interaction angle.

noise and vibration produced. Subsequently, circulation strength and trajectory of the vortex segment may be influenced.

3. The miss distance between a vortex segment and the oncoming rotor blade can enhance a BVI event as the miss distance becomes smaller.

4. The interaction angle between the vortex segment and blade in the plane of the rotor (whether an interaction is parallel or not) can alter both the magnitude of BVI noise and the propagation efficiency.

Active control has the potential to mitigate BVI noise and vibration by modifying any of the three characteristics affecting BVI strength: pressure fluctuations, miss distance or interaction angle. Both active and passive techniques have been developed for vibration and noise reduction, and it is likely that the best rotor could benefit from a judicious combination of these two techniques. However, this paper will focus primarily on active techniques. A number of active control approaches, illustrated schematically in Fig. 2, have been developed for vibration reduction. ${ }^{1,2}$ These fall into one of two categories: (a) active control approaches aimed at reducing vibrations in the rotor before they propagate into the fuselage, and (b) active control approaches implemented in the fuselage using an approach known as active control of structural response (ACSR). Within the first category of active control, where the primary objective is to reduce vibrations in the rotor, two approaches have emerged. These are (1) higher harmonic control (HHC) where the blades are activated in the non-rotating swashplate by introducing pitch commands, and (2) individual blade control (IBC) where each blade can be controlled independently in the rotating frame. Several implementations of IBC are available: (i) the conventional or earliest implementation based on pitch actuation at the blade root in the rotating system, (ii) actively controlled partial-span trailing-edge flaps, and (iii) the active-twist rotor where the entire blade is twisted by piezoelectric fiber embedded in the blade. Additional descriptions of these approaches can be found in Refs. 1 and 2.

During the last decade, the HHC and IBC approaches, developed primarily for vibration reduction, have also been considered as a means of reducing BVI noise. However, the control algorithms used are essentially the same as those devised for vibration reduction, and no attempts were made to develop special algorithms for the noise reduction problem.

Among these active control approaches, HHC and conventional IBC were developed earlier and have been tested extensively in wind tunnels and flight tests. Excellent vibration reduction of more than $80 \%$ has been demonstrated using these approaches. The HHC and IBC approaches have also been explored for BVI noise reduction..$^{4-8}$ The HART (higher-harmonic-control aeroacoustics rotor test) study ${ }^{7}$ conducted in the GermanDutch Wind Tunnel (DNW) is especially notable, where the important parameters associated with BVI noise generation such as wake geometry, blade loading, rotor dynamics, etc. as well as the effects of HHC inputs have been studied systematically. The results obtained in the HART provided invaluable insight into the physical mechanisms of BVI noise and improved BVI noise modeling significantly by serving as an excellent validation basis. Maximum noise reduction of $6 \mathrm{~dB}$ was found using open loop HHC control in the HART study, 


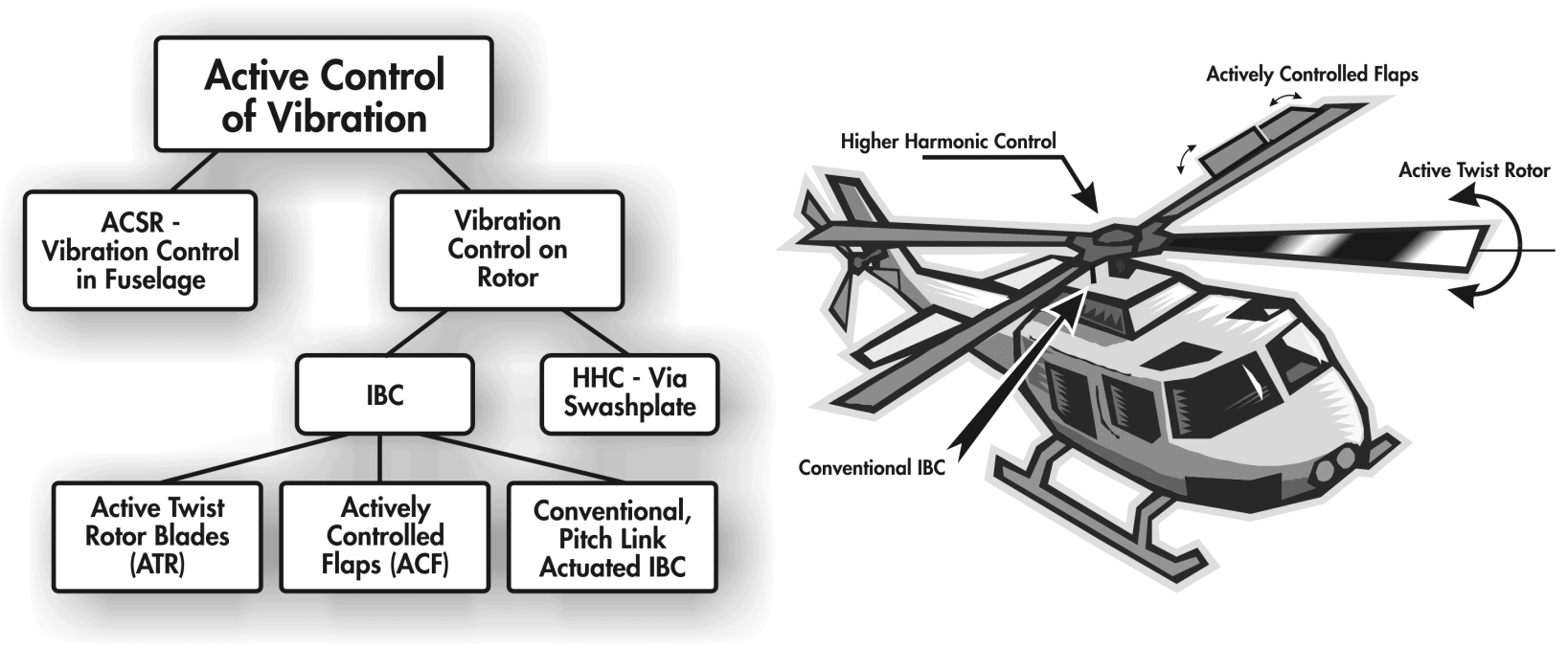

Figure 2. An Overview of Active Control Techniques.

which is comparable to other studies using the conventional IBC approach. ${ }^{9-12}$ It is noteworthy that although most noise reduction studies thus far have been conducted in open loop, a recent flight test with a BO-105 helicopter in Europe has demonstrated a closed-loop IBC controller using on-board microphones. ${ }^{13,14}$

More recently, actively controlled flaps have emerged as an efficient means of the active control of vibrations due to BVI as well as the alleviation of dynamic stall induced vibrations. ${ }^{1,15-21}$ These studies on ACF system implemented in both single and dual flap configurations have demonstrated vibration reduction comparable to those achieved with HHC or conventional IBC, with no adverse effects on helicopter airworthiness and significantly less power requirement as compared to the blade root actuation approaches. ${ }^{1}$ Wind tunnel tests have also shown the feasibility of the ACF for vibration reduction. ${ }^{21,22}$ During the Smart Material Actuated Rotor Technology (SMART) program conducted by Boeing, ${ }^{23,24}$ a full scale piezoelectrically actuated flap system for vibration and noise control for a five-bladed bearingless MD-900 rotor, was tested on a whirl tower, to demonstrate control effectiveness. Two recent papers have focused specifically on the consequences of vibration reduction on noise levels and the effects of noise reduction on vibration ${ }^{25}$ as well as simultaneous vibration and noise reduction. ${ }^{26}$ The present paper combines and extends the research described in the previous papers ${ }^{25,26}$ while focusing on important issues dealing with the practical implementation of single and dual ACF systems.

The current paper has several objectives:

1. Explore the potential of BVI noise reduction and simultaneous vibration and noise reduction using the ACF approach.

2. Determine and compare the effectiveness of the ACF in the closed loop mode for noise and vibration reduction on two different rotor configurations, namely, a four-bladed MBB BO-105 hingeless rotor and a five-bladed MD-900 bearingless rotor.

3. Evaluate the effectiveness of passive methods on the vibration and noise reduction using advanced geometry tips with anhedral and dihedral, and compare them with the active approach.

4. Examine a number of practical implementation issues associated with the ACF system, such as the effects of practical saturation limits, constant and 1/rev pitch inputs, and flap overhang.

These goals allow a realistic assessment of the potential of the ACF approach for BVI noise reduction and the simultaneous control of both vibration and noise.

\section{Mathematical Model}

The mathematical model of the actively controlled rotor/flap system consists of several fairly complex ingredients which are combined into a sophisticated aeroelastic response and noise generation simulation that 
is used to demonstrate active vibration and noise reduction using single and dual ACF systems implemented on two different rotor configurations. The principal ingredients of this simulation are: (1) a structural dynamic model; (2) an aerodynamic model; (3) an acoustic model and (4) a coupled trim/aeroelastic solution procedure that generates the blade response. The various ingredients of this model are described concisely in the following subsections.

\section{A. Structural Dynamic Model}

Two rotor configurations are considered in the present study, as illustrated in Fig. 3. The first configuration is described extensively in Refs. 15 and 27, and it represents a four-bladed hingeless rotor shown in Fig. 3a that resembles a MBB BO-105 rotor. Each blade has a root offset $e$, and it rotates with constant angular speed $\Omega$, and has fully-coupled flap, lead-lag, and torsional dynamics including nonlinearities due to moderate blade deflections. The equations of motion are discretized using the global Galerkin method, based upon the free vibration modes of the rotating blade. Three flapping modes, two lead-lag modes and two torsional modes are used in the actual implementation.

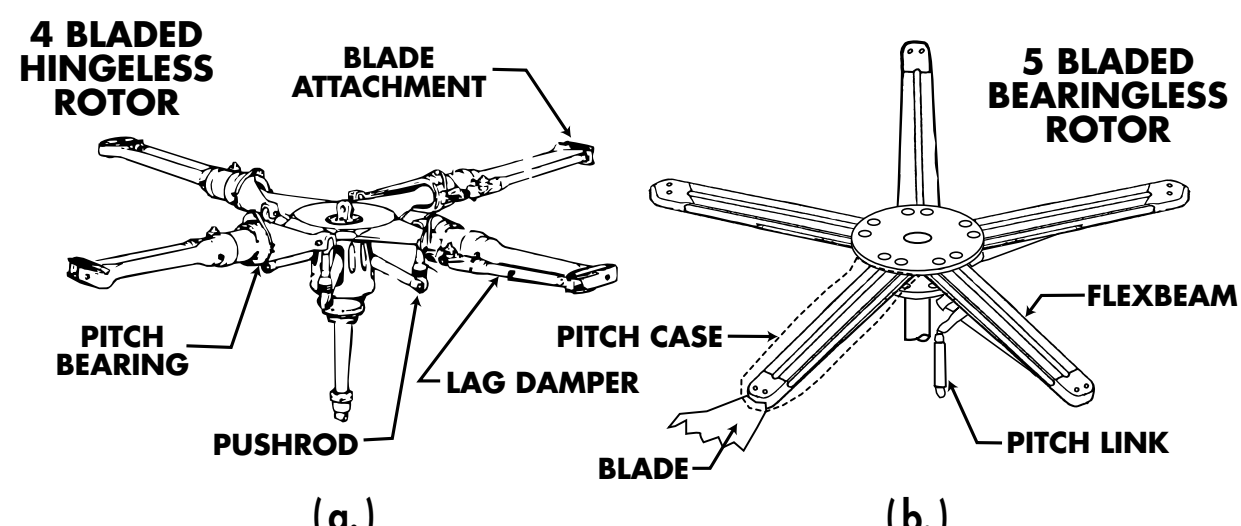

Figure 3. Hingeless and bearingless rotor hubs.

The second configuration is a five-bladed bearingless rotor that resembles the MD-900 Explorer rotor. This rotor was used in the Boeing smart material actuated rotor technology (SMART) tests. ${ }^{24}$ The structural dynamic model is based on a composite blade model developed by Yuan and Friedmann ${ }^{28}$ which is capable of modeling transverse shear deformations, cross sectional warping and swept tips. The equations of motion are formulated using a finite element discretization of Hamilton's principle and assuming moderate deflections. The blade is discretized by beam type elements, having 23 nodal degrees of freedom. Originally the model was limited to modeling hingeless blades. In the present study the structural dynamic model was modified and extended to properly represent multiple load paths by including the pitchcase elements that are an important characteristic of bearingless rotor design. The resulting finite element model obtained after this modification is quite similar to the bearingless rotor configuration modeled with the RCAS code (Rotorcraft Comprehensive Analysis System) which is a comprehensive rotorcraft analysis system; also based on the finite element method. ${ }^{29}$ Since our structural dynamic model and the RCAS model are quite similar, the bearingless rotor blade model shown in Fig. 4 captures the essence of both models. The RCAS model was used to correlate the natural frequencies with the results obtained by the current analysis in the validation section that is presented next. The snubber spring and damper elements in the RCAS model are replaced by concentrated stiffness and damping at the blade root, while the control slide is not included in the current analysis.

The flexbeam portion of the rotor hub is modeled by beam elements that are cantilevered to the hub at node number 2, as shown in Fig. 4. The pitchcase is also modeled by beam-type elements, forming a second load path. The inboard end node (node 31) of the pitchcase is restrained to the hub through the snubber-damper assembly, modeled by linear lead-lag and flap springs, and a lead-lag damper, with spring constants and damping coefficients determined experimentally. This node is also connected to the pitch link, where the pitch control is applied. At the blade-flexbeam-pitchcase junction (node 5), continuity is imposed for both displacements and slopes for flap and lead-lag bending as well as displacements in the other degrees of freedom. 


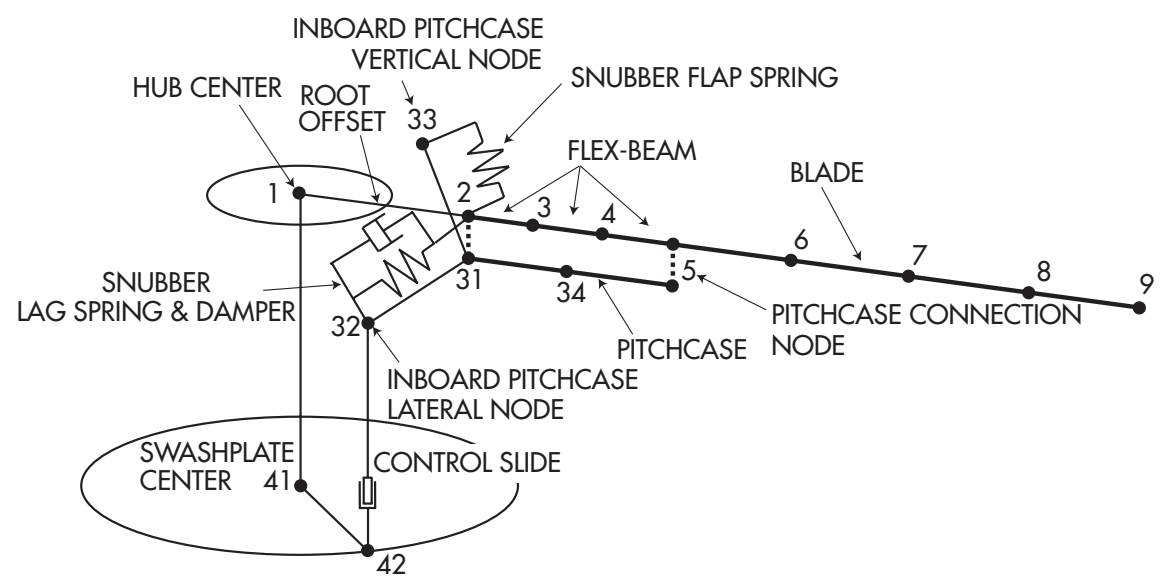

Figure 4. RCAS finite element model for a bearingless rotor.

Note that the bearingless blade model includes multiple load paths associated with beam-type elements for the pitchcase, and continuity is enforced at the blade/pitchcase junction. In the actual MD-900 model, 12 spatial elements are used to model the flexbeam/blade combination, and 2 elements model the pitchcase. Modal reduction with eight coupled natural rotating modes (four flap, two lead-lag, one torsion and one axial) is used to solve the aeroelastic equations of motion.

\section{B. Aerodynamic Model}

The aerodynamic model consists of several important ingredients that are essential for a coupled aeroelastic/acoustic simulation. These are:

1. An unsteady comprehensive, two-dimensional aerodynamic model for the blade-flap combination that accounts for variations in the oncoming velocity. The model is based upon a rational function approximation (RFA) of the aerodynamic loads, ${ }^{16}$ which produces aerodynamic cross-sectional loads (lift, moment, flap-hinge moment) in the time domain.

2. An extension of the RFA model used for the computation of the chordwise pressure distribution, ${ }^{25}$ that is required for the acoustic calculations.

3. An enhanced free wake model that provides the non-uniform inflow distribution at closely spaced azimuthal steps, together with a dual trailing vortex structure needed for the BVI noise calculations. ${ }^{25,30,31}$ The enhancements consist of improved wake resolution and refined modeling of the inboard wake structure which contributes to elevated noise levels that has been observed in experiments. ${ }^{7}$

4. A simple, linear drag model that accounts for additional drag due to flap deflection, that increases the power required for the rotor to operate, as described in Ref. 32.

\section{Acoustic Model}

The acoustic analysis is based on a modified version of the WOPWOP ${ }^{33}$ code which computes helicopter noise using the Ffowcs-Williams Hawkings equation with the quadrupole term neglected. In the current research the WOPWOP code was modified so that now it is based on a fully flexible blade model that is consistent with the structural dynamic model described in a previous subsection (A). The input into the acoustic code is the unsteady pressure distribution on the surface of the blade that is calculated from a combination of the RFA unsteady aerodynamic model which yields chordwise and spanwise pressure distributions that also depend on the non-uniform inflow provided by the free-wake model. 


\section{Solution Procedure}

The combined structural and aerodynamic equations form a system of coupled differential equations that can be cast in state variable form. They are then integrated in the time domain using the Adams-Bashforth DE/STEP predictor-corrector algorithm. Two types of trim procedures can be implemented, including a propulsive trim where six equilibrium equations (three forces and three moments) are enforced, as well as a wind tunnel trim where zero pitch and moment conditions are enforced. ${ }^{26}$ These trim equations are solved in a coupled manner with the aeroelastic equations of motion. Hub vibratory loads are obtained by integrating the distributed aerodynamic and inertial loads over the blades. The BVI noise is computed by the acoustic module using the unsteady distributed aerodynamic pressure and blade response which are obtained after the coupled trim/aeroelastic calculation is completed.

\section{Higher Harmonic Control Algorithm for Vibration and Noise Reduction}

The higher-harmonic control algorithm is used for both noise and vibration reduction. This algorithm has been the subject of a recent paper, ${ }^{34}$ wherein the stability, robustness, and convergence properties of the algorithm together with a number of variants are addressed in detail.

The algorithm is based on a linear, quasi-static, frequency domain representation of helicopter response to control inputs. The inputs to the algorithm are comprised of a combination of flap deflection harmonics with discrete frequencies of $N_{\min }-N_{\max } /$ rev. The total flap deflection is given as

$$
\delta(\psi)=\sum_{N=N_{\min }}^{N_{\max }}\left[\delta_{N c} \cos (N \psi)+\delta_{N s} \sin (N \psi)\right]
$$

The lowest component of higher harmonic frequency input has been typically chosen to be $2 /$ rev. However, in the current paper the effects of constant $(0 / \mathrm{rev})$ and $1 / \mathrm{rev}$ inputs are also discussed. The choice of $N_{\max }$ is based on the number of the blades, since the dominant vibrations at the hub are $N_{b} /$ rev. Therefore, in the studies of the four-bladed BO-105 rotor $N_{\max }=5$ and in the five-bladed MD-900 rotor $N_{\max }=6$. These pitch deflections are related to the vibration or noise level magnitudes through a transfer matrix $\mathbf{T}$, given by

$$
\mathbf{T}=\frac{\partial \mathbf{z}_{k}}{\partial \mathbf{u}_{k}} .
$$

The control strategy is based on the minimization of a performance index that was originally developed for vibration reduction ${ }^{35}$ which is a quadratic function of the quantities that are being reduced (vibration or noise) $\mathbf{z}_{k}$ and control input amplitudes $\mathbf{u}_{k}$ :

$$
J\left(\mathbf{z}_{k}, \mathbf{u}_{k}\right)=\mathbf{z}_{k}^{\mathrm{T}} \mathbf{Q} \mathbf{z}_{k}+\mathbf{u}_{k}^{\mathrm{T}} \mathbf{R} \mathbf{u}_{k},
$$

The subscript $k$ refers to the $k^{\text {th }}$ control step, reflecting the discrete-time nature of the control. The time interval between each control step must be sufficient to allow the system to return to the steady state, typically in $3-5$ revolutions, so that the vibration or noise levels can be accurately measured. The optimal control law is given by:

$$
\mathbf{u}_{k, \text { opt }}=-\mathbf{D}^{-1} \mathbf{T}^{\mathrm{T}} \mathbf{Q}\left\{\mathbf{z}_{0}-\mathbf{T} \mathbf{u}_{0}\right\}
$$

where

$$
\mathbf{D}=\mathbf{T}^{\mathrm{T}} \mathbf{Q T}+\mathbf{R}
$$

For a well-identified linear system the algorithm converges to the optimum value in a single step. ${ }^{34}$ However, if the helicopter cannot be perfectly represented by a linear model, the optimal value will not be reached after the first step. The convergence issue of the algorithm was addressed in Ref. 34, where a relaxed version of the algorithm, described concisely below, was developed. Traditionally, the control input update is represented in iterative form as shown in Eq. (6):

$$
\mathbf{u}_{\mathrm{k}+1}=\mathbf{u}_{\mathrm{k}}+\Delta \mathbf{u}_{\mathrm{k}} .
$$

In the relaxed variant of the algorithm, a relaxation factor $\alpha_{R}$ is introduced,

$$
\mathbf{u}_{\mathrm{k}+1}=\mathbf{u}_{\mathrm{k}}+\alpha_{R} \Delta \mathbf{u}_{\mathrm{k}},
$$


where $0<\alpha_{R}<1$. This has been shown to increase the robustness of the algorithm at the expense of convergence speed. ${ }^{34}$ An adaptive version ${ }^{34,35}$ of the HHC algorithm was also useful in the noise reduction studies. In the adaptive variant, the transfer matrix $\mathbf{T}$ is identified online, using a recursive least-squares technique, following the method described in Ref. 34 .

For vibration reduction (VR) studies, the vector $\mathbf{z}_{k}$ consists of $N_{b} /$ rev vibration levels as represented by hub shears and moments, given in Eq. (8),

$$
\mathbf{z}_{k, \mathrm{VR}}=\left[\begin{array}{c}
F_{H X} \\
F_{H Y} \\
F_{H Z} \\
M_{H X} \\
M_{H Y} \\
M_{H Z}
\end{array}\right]
$$

When the controller is used for BVI noise reduction the vibration levels objective vector $\mathbf{z}_{k, \mathrm{VR}}$ is replaced by the $6^{\text {th }}-17^{\text {th }}$ harmonic components of BVI noise as given in Eq. (9), which are measured by a microphone installed at a suitable location. As shown in Fig. 5, these locations can be located on the skid or landing gear of the helicopter. A flight test ${ }^{36}$ has suggested that, for many flight conditions, skid-mounted microphones provide good correlation with ground-based noise levels. Furthermore, previous studies by the authors ${ }^{26,37}$ have shown that feedback microphones placed on the right landing skid at the rear (but not on the left skid), as illustrated in Fig. 5 labeled SKID-REAR, correlate well with noise levels on a carpet plane below the rotor. Therefore, in the present study the feedback microphone is placed on the SKID-REAR location.

$$
\mathbf{z}_{k, \mathrm{NR}}=\left[\begin{array}{c}
N_{H 06} \\
N_{H 07} \\
N_{H 08} \\
\vdots \\
N_{H 17}
\end{array}\right]
$$

Thus, the noise control law is identical to the control law used for vibration reduction except that the objective function $J$ consists of the $6^{\text {th }}-17^{\text {th }}$ blade passage frequency harmonic components of acoustic pressure (the most significant part of BVI noise) in quadratic form.

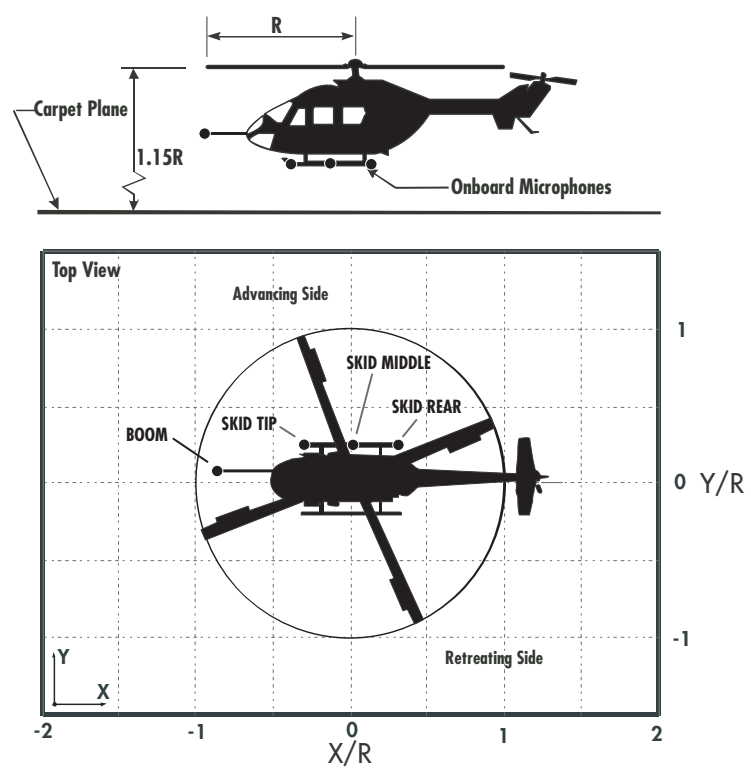

Figure 5. Microphone locations on and around the helicopter for noise feedback. 
For simultaneous reduction (SR) problems, a combined output vector is defined

$$
\mathbf{z}_{k, \mathrm{SR}}=\left[\frac{\mathbf{z}_{k, \mathrm{VR}}}{\mathbf{z}_{k, \mathrm{NR}}}\right] .
$$

Where the vector $\mathbf{z}_{k, \mathrm{SR}}$ is simply a partitioned combination of hub shear and noise levels. The weighting matrix $\mathbf{Q}$ is used to adjust the control effort so as to achieve a desirable balance between the vibration levels and noise harmonic reductions.

If the controller requires large flap deflections exceeding the practical limits of the flap actuation system, the flap may saturate and lose its effectiveness. Therefore in this study, for the practical implementation of an ACF system, flap deflections will be limited to values that do not exceed $\delta_{\max }=4^{\circ}$. The HHC algorithm has been modified to account for actuator saturation as described in Ref. 38.

\section{Model Validation}

An extensive structural, aerodynamic, and acoustic validation study was previously conducted using $\mathrm{HART}^{7}$ data, and is documented in Refs. 25 and 26. Overall, very good correlation was achieved, featuring remarkable reproduction of acoustic signatures at locations around the rotor for the baseline case. This validation effort was for a $40 \%$ scaled MBB BO-105 hingeless rotor. In this section, the bearingless rotor structural model, resembling the MD-900 SMART rotor, is correlated with results generated by two comprehensive rotor codes.

The natural frequencies of the linear, undamped system in a vacuum are first calculated and compared to those obtained using RCAS, as shown in Table 1. The corresponding normal mode shapes are compared in Fig. 6. The comparisons are quite good.

Table 1. Comparison of natural frequencies for MD-900 bearingless main rotor.

\begin{tabular}{c|cc} 
& Current Simulation & RCAS \\
\hline$\omega_{L 1}$ & 0.654 & 0.654 \\
$\omega_{F 1}$ & 1.043 & 1.048 \\
$\omega_{F 2}$ & 2.573 & 2.572 \\
$\omega_{L 2}$ & 3.488 & 3.498 \\
$\omega_{F 3}$ & 4.472 & 4.473 \\
$\omega_{T 1}$ & 5.667 & 5.409 \\
$\omega_{F 4}$ & 7.270 & 7.273 \\
$\omega_{A 1}$ & 25.70 & 25.82
\end{tabular}

In order to validate both the blade response and flap effectiveness of the bearingless rotor, predictions of tip pitch deflection and blade torsional moment are calculated. The results generated by the simulation for a baseline (no control) case and an active control case with prescribed flap deflections are compared against the results obtained using the comprehensive rotorcraft code CAMRAD II. ${ }^{20,39}$ The baseline case simulates descending flight condition at $\mu=0.2$, with a thrust coefficient of $C_{T} / \sigma=0.0774$. The rotor is trimmed to zero first-harmonic blade flapping and the specified $C_{T} / \sigma$ using a wind-tunnel type trim procedure. ${ }^{26,27}$ For the controlled case, the flap deflection is prescribed at a single frequency of $4 / \mathrm{rev}$ with a maximum $2^{\circ}$ deflection amplitude, $\delta_{f}=2 \cos (4 \psi-240)$.

The blade tip pitch response (torsion, collective, and cyclic, but excluding built-in pretwist) for the baseline and controlled cases are compared in Fig. 7a. The general trends of pitch variation with azimuth for both the baseline and controlled cases are quite similar, except that the current simulation exhibits a nearly constant offset angle from the CAMRAD II predictions. This difference may be attributed to the fact that the cambered airfoils used on the actual MD-900 rotor and in the CAMRAD II predictions are replaced by a symmetric airfoil in the present simulation. Note that the $2^{\circ}$ of prescribed flap deflection induces roughly the same magnitude of total blade tip pitch deflection in the CAMRAD II prediction and the current simulation.

Blade torsional moments at a 40\% radial location are compared in Fig. 7b, with the same prescribed flap deflection. The predictions of current simulation agrees reasonably well with that of CAMRAD II. 


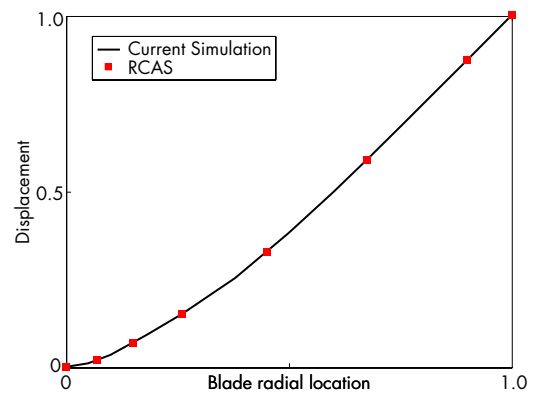

(a) 1st lead-lag mode

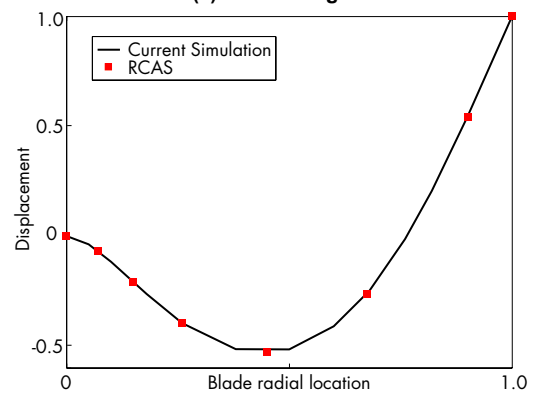

(c) 2nd flap mode

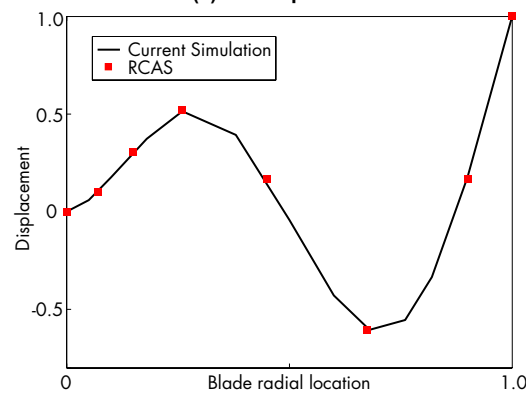

(e) 3rd flap mode

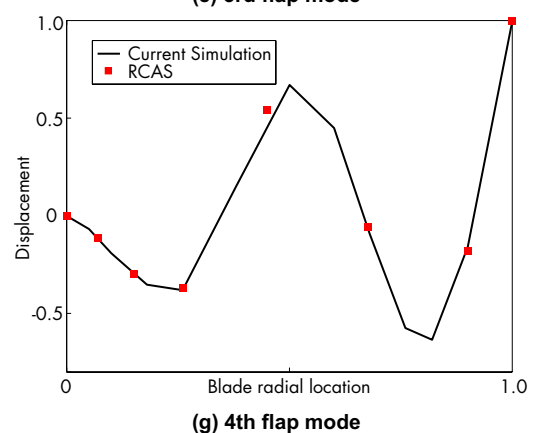

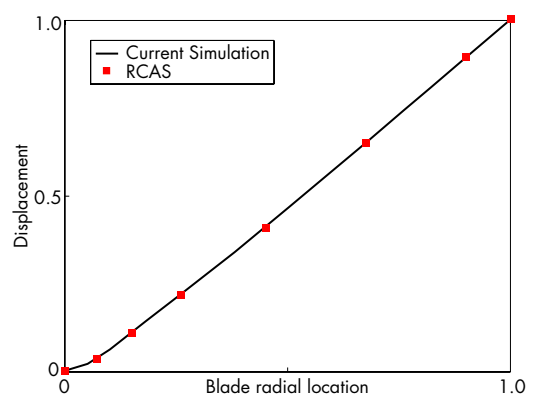

(b) 1st flap mode

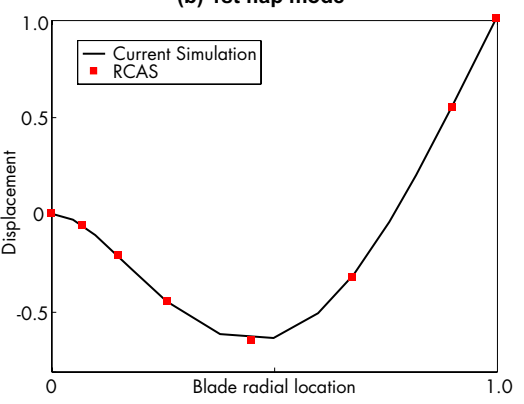

(d) 2nd lead-lag mode

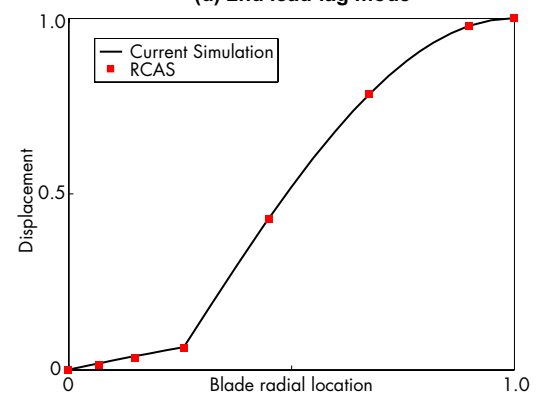

(f) 1st torsion mode

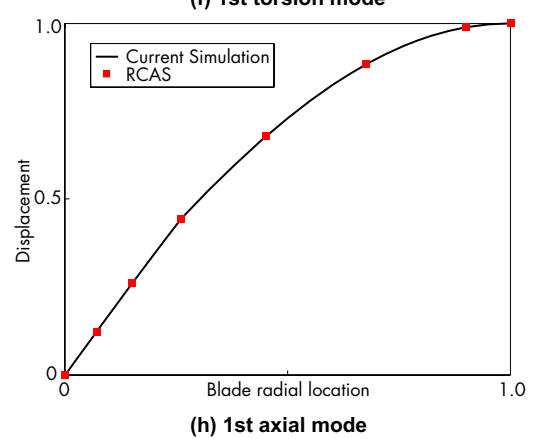

Figure 6. Comparison of blade normal modes for MD-900 bearingless main rotor. 


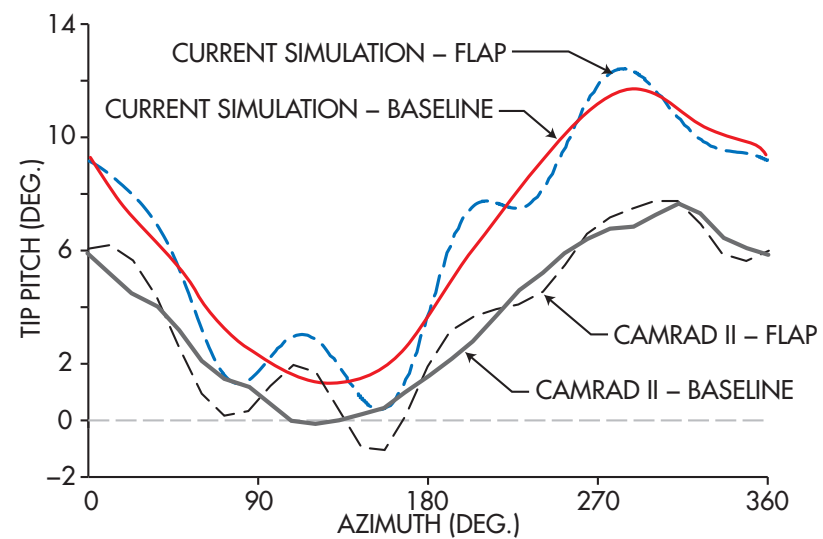

(a.)

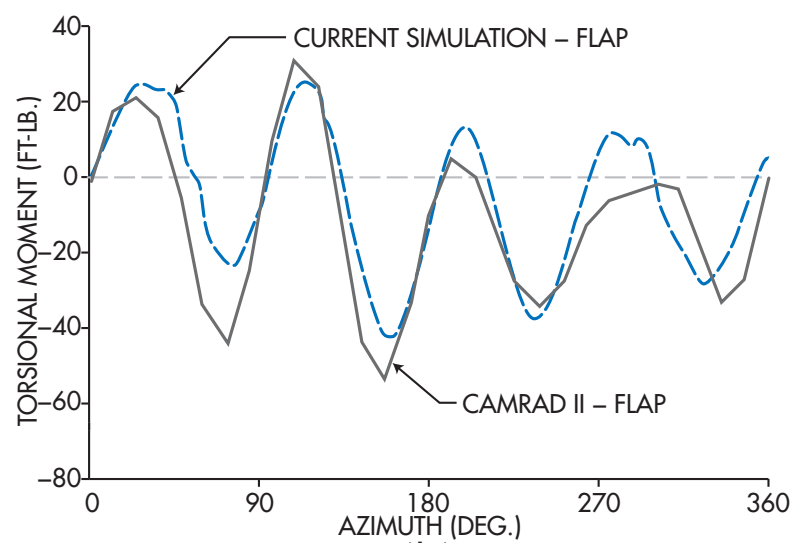

(b.)

Figure 7. Simulation correlation with CAMRAD II.

\section{Results for MBB BO-105 Hingeless Rotor}

The results presented in this section were obtained for a helicopter configuration resembling a fullscale MBB BO-105 helicopter with a four-bladed hingeless rotor system. The properties of the helicopter configuration used in the computations are summarized in Table 2. The characteristics of the actively controlled flap configurations are given in Table 3, including a single servo flap configuration and a dual servo flap configuration. As mentioned earlier, a microphone is placed on the SKID-REAR location for noise feedback control, as illustrated in Fig. 5. All vibration, noise and simultaneous reduction studies were performed at the same flight condition, a simulated $6.5^{\circ}$ descent in heavy BVI at $\mu=0.15$.

Table 2. MBB BO-105 hingeless blade configuration.

\begin{tabular}{ll}
\hline$\underline{\text { Rotor Data }}$ & \\
$N_{b}=4$ & $c=0.05498 L_{b}$ \\
$\omega_{F}=1.12,3.41,7.62$ & $C_{d o}=0.01$ \\
$\omega_{L}=0.73,4.46$ & $C_{m o}=0.0$ \\
$\omega_{T}=3.17$ & $a_{o}=2 \pi$ \\
$\theta_{\mathrm{tw}}=-8^{\circ}$ & $\theta_{\mathrm{FP}}=6.5^{\circ}$ \\
$\gamma=5.5$ & $\sigma=0.07$ \\
$\beta_{p}=2.5^{\circ}$ & \\
Helicopter Data & \\
\hline$C_{W}=0.005$ & $\mu=0.15$ \\
$L_{b}=4.91 \mathrm{~m}$ & $M_{\mathrm{ref}}=53 \mathrm{~kg}$ \\
$\Omega=425 \mathrm{RPM}$ & \\
\hline
\end{tabular}

Table 3. Flap configuration.

\begin{tabular}{ll}
\hline$c_{c}=0.25 c$ & \\
$\frac{\text { Single Servo Flap }}{x_{c}=0.75 L_{b}}$ & $L_{c}=0.12 L_{b}$ \\
Dual Servo Flap & \\
\hline$x_{c}^{1}=0.72 L_{b}$ & $L_{c}^{1}=0.06 L_{b}$ \\
$x_{c}^{2}=0.92 L_{b}$ & $L_{c}^{2}=0.06 L_{b}$ \\
\hline
\end{tabular}

\section{A. Vibration Reduction}

The active flaps are first used for vibration reduction, with $4^{\circ}$ saturation limits imposed. The results for the baseline and controlled 4/rev vibratory loads are shown in Fig. 8. The dual flap configuration can achieve vibration reduction of $68 \%$ with saturation limits imposed, while the less effective single flap achieves a reduction of $35 \%$. The overall vibration objective functions are reduced by $46 \%$ and $86 \%$ for single and dual flap configurations, respectively.

The noise footprints on a carpet plane located 1.15 radii below the hub for the baseline, single flap, and dual flap configurations are shown in Fig. 9. The sound pressure level (SPL) in decibel (dB) is computed with respect to a reference pressure of $20 \mu \mathrm{Pa}$. The noise directivity of the baseline case is characterized by the high noise levels on the advancing and retreating side. The noise levels have a slight $1 \mathrm{~dB}$ noise increase 


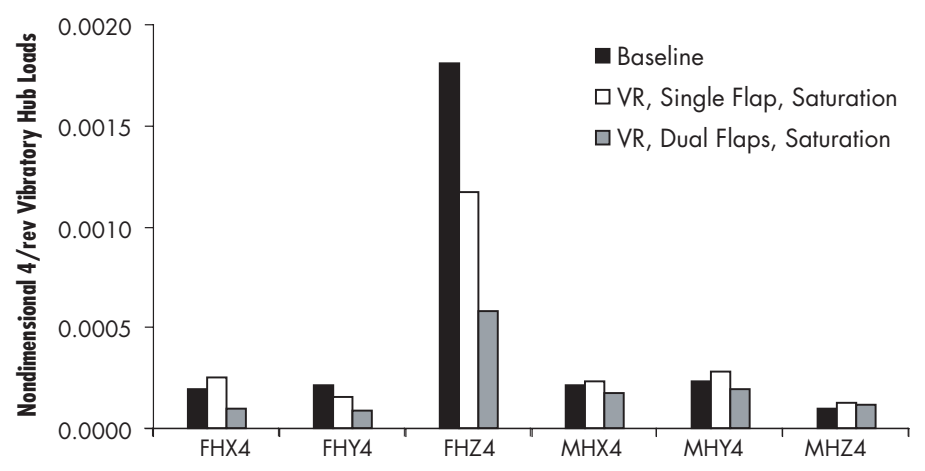

Figure 8. Vibration levels and its reduction with 1 and 2 flaps, full-scale BO-105.

on the retreating side as shown in Fig. 9. This suggests that deflection-limited actively controlled flaps may be used to reduce vibration without a significant effect on noise. This behavior differs significantly from that observed for HHC and IBC configurations. ${ }^{5,9}$
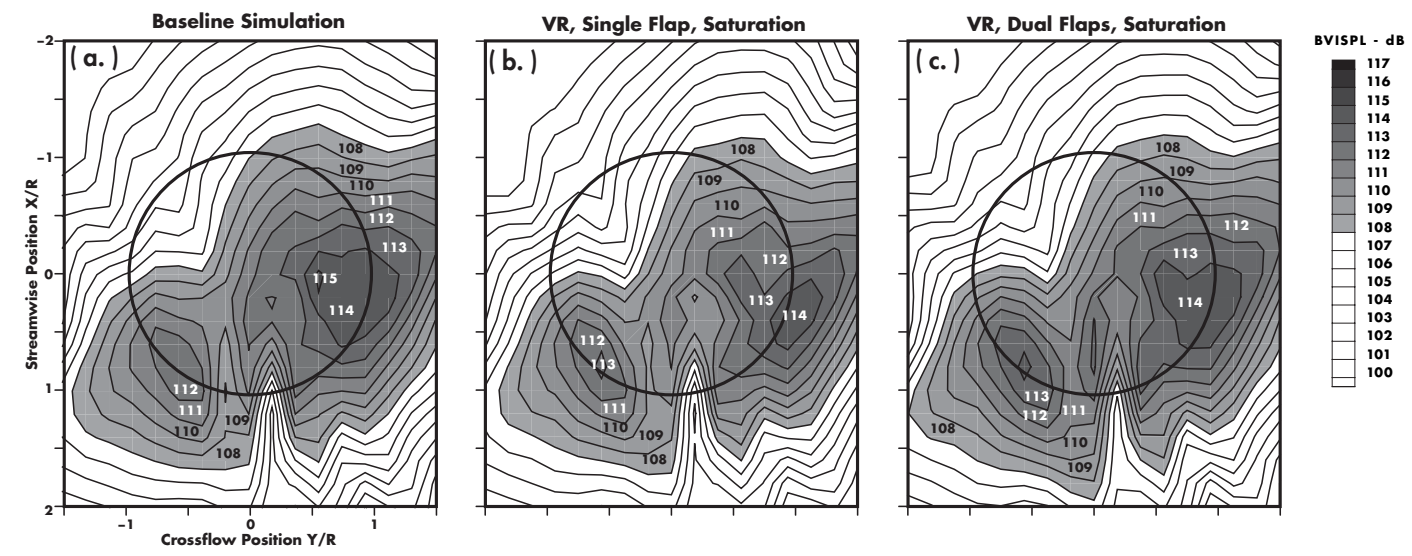

Figure 9. Noise levels during vibration reduction with 1 and 2 flaps, full-scale BO-105.

The deflections of the actively controlled flap over a rotor revolution for the single and dual ACF configurations are shown in Fig. 10. The maximum flap deflection is less than $4^{\circ}$ with saturation limits implemented. It is interesting to note that the time history of the flap deflection for the outboard flap differs substantially from the inboard flap, particularly in the retreating blade region.
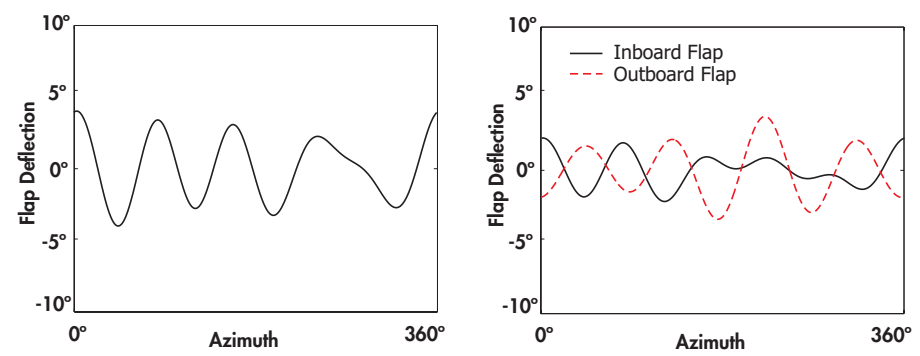

Figure 10. Flap deflections, vibration reduction.

\section{B. BVI Noise Reduction}

The adaptive variant of the HHC controller was implemented for noise reduction using the feedback microphone SKID-REAR. The adaptive controller has been identified to have significant advantages over the 
conventional HHC algorithm for active noise control. ${ }^{26}$ The adaptive controller was also implemented with $4^{\circ}$ saturation limits imposed. The noise level at SKID-REAR was reduced by $5 \mathrm{~dB}$ with single flap and $6 \mathrm{~dB}$ with dual flap configuration. The resulting noise levels on the carpet plane are shown in Figs. 11b-11c. Both single and dual flap configurations are found to be effective in reducing the advancing side noise on the carpet plane. The reductions range from $3-5 \mathrm{~dB}$ as shown in Fig. 11. However increases of $1-2 \mathrm{~dB}$ for these cases are observed on the retreating side.
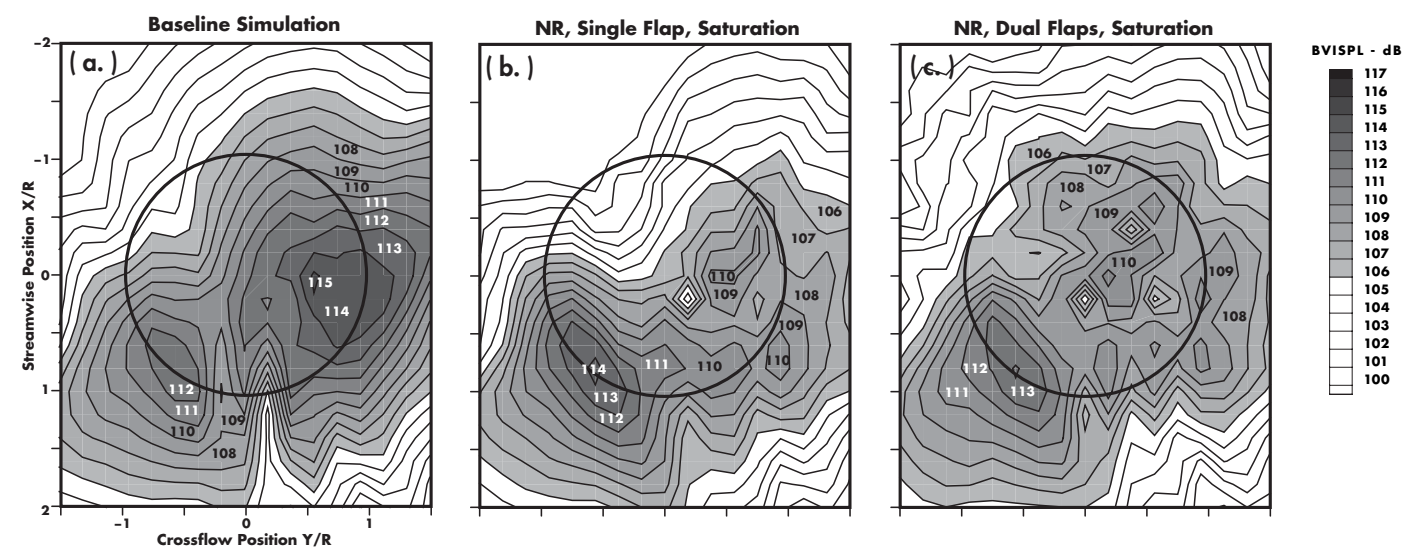

Figure 11. Noise reduction with 1 and 2 flaps, adaptive HHC.

The vibration levels, shown in Fig. 12, were also monitored during the active noise reduction process. It can be seen in Fig. 12 that noise reduction with the dual flap configuration is associated with vibration penalty, where the overall vibration objective is increased by $130 \%$. In the single flap case, the vibration objective function remains unchanged during the active noise control. However, as evident from Fig. 12, the vertical vibratory hub shear FHz4 is always reduced. The flap deflections for noise reduction are shown in Fig. 13.

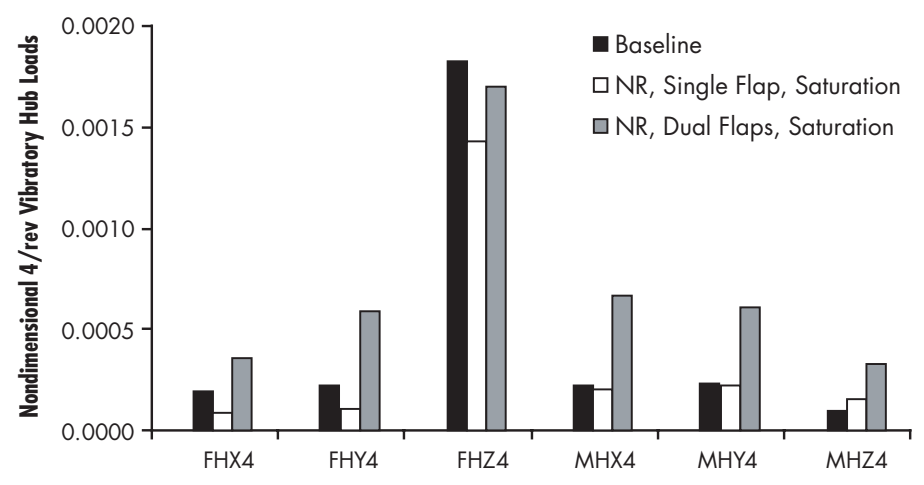

Figure 12. Vibration levels, noise reduction with 1 and 2 flaps, adaptive HHC.
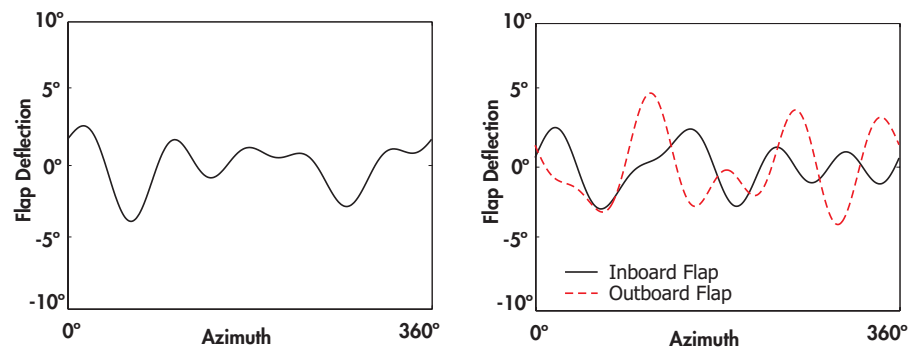

Figure 13. Flap deflections, noise reduction. 
The results shown have demonstrated that active noise reduction by means of actively controlled flaps with saturation limits is an effective and practical option. Good correlation has been found between the BVI noise at the skid-mounted microphone SKID-REAR and the carpet plane below the helicopter, however, it was also noted that both flap configurations considered experienced slight increases in retreating side noise while the advancing side noise was reduced. This is due to the location of the feedback microphone, SKID-REAR, on the advancing side. It is also found that poor correlation exists between retreating side noise and a microphone on the left skid at the rear, suggesting the increased complexity of the noise radiation pattern on the retreating side. ${ }^{40}$

\section{Simultaneous Vibration and Noise Reduction}

Active control using the adaptive HHC algorithm was also implemented for the simultaneous reduction of vibration and noise, with $4^{\circ}$ saturation limits imposed. The reductions in vibration levels for single or dual flap configuration are shown in Fig. 14. The dominant vertical hub shears were reduced by about $40 \%$.

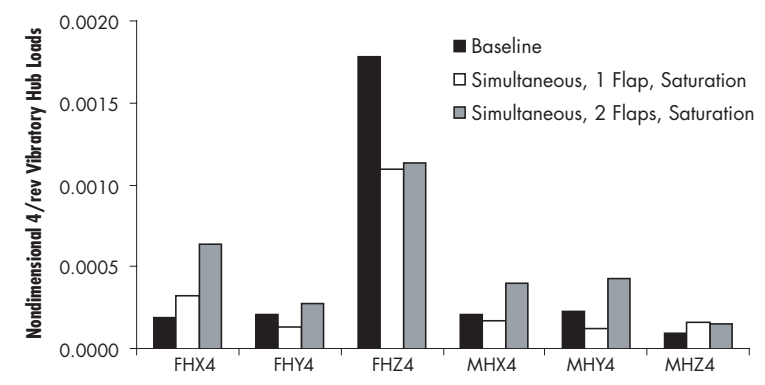

Figure 14. Vibration levels showing reduction from baseline, simultaneous reduction with 1 and 2 flaps.

The noise levels on the carpet plane are shown in Fig. 15. Noise reductions of $3-5 \mathrm{~dB}$ are found on the advancing side, with slight penalties on the retreating side. By comparison the dual flap configuration is more effective for noise reduction. The flap deflections for simultaneous noise and vibration reduction are shown in Fig. 16 for the single and dual ACF configurations. The maximum flap deflection is $4^{\circ}$.
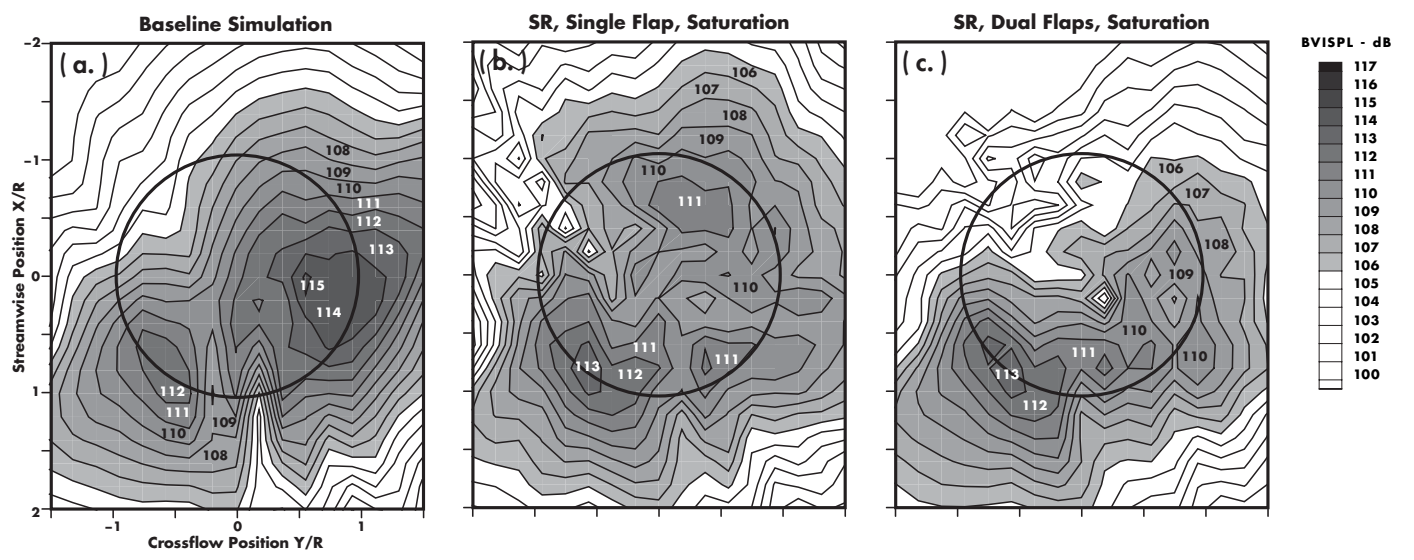

Figure 15. Noise carpet plot showing reduction from baseline, simultaneous reduction with 1 and 2 flaps.

The results have demonstrated that simultaneous active reduction of noise and vibration with actively controlled flaps is feasible.

\section{The Effects of Constant and 1/rev Pitch Inputs}

For the active control studies of noise and vibration explored thus far, the flap control harmonics have been taken to be a combination of $2-5 / \mathrm{rev}$, which are the harmonic components useful for reducing vibration and noise in four-bladed rotors. Furthermore, recent studies ${ }^{27}$ by the authors have determined that the effects of flap inputs on the rotor trim are small, especially in the cases when the flap saturation is imposed. 

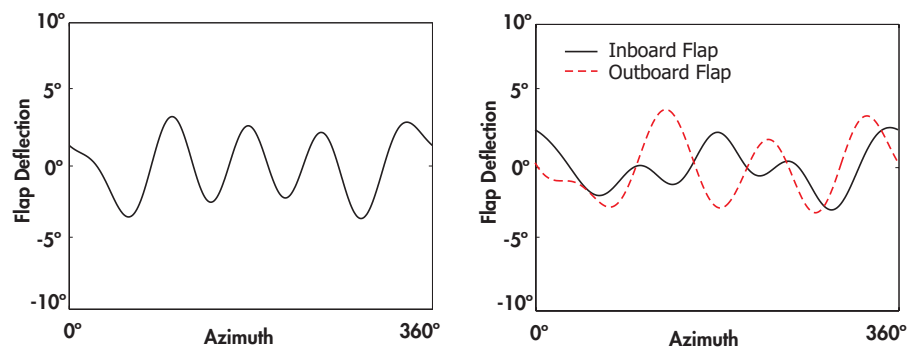

Figure 16. Flap deflections, simultaneous reduction.

In a recent paper ${ }^{41}$ the possibility of BVI noise mitigation has been raised using a steady flap deflection. Steady flap deflections are simple to implement and have obvious benefits. Reference 41 indicated that noise reduction by approximately $6 \mathrm{~dB}$ under BVI condition may be possible, with a servo flap inboard of $70 \%$ radial location. It should be noted that the length of the flap extended over more than $50 \%$ of the blade radius, and the maximum flap deflection used was $20^{\circ}$. Clearly such flap systems differ drastically from the active flap employed in the present study, both in size as well as maximum flap deflections. Nevertheless, it was deemed interesting to examine the effects of constant and 1/rev pitch inputs on BVI noise, using active flap configurations employed in the present study.

In this section, the control input frequencies were expanded to include the $0 / \mathrm{rev}$ or $1 / \mathrm{rev}$ components, otherwise the control algorithm for noise reduction is the adaptive algorithm as described previously, with $4^{\circ}$ saturation limits. The BVI noise contours are shown in Fig. 17 , with $0 /$ rev or $1 /$ rev inputs added to the $2-5 /$ rev components. The reductions on the advancing side are comparable to the amount achieved by using 2-5/rev components only. However, it appears that the inclusion of contant and $1 /$ rev inputs has some advantage on the retreating side, where the BVI noise is not increased unlike those shown in Fig. 11. Interestingly, the single flap configuration seems to have an advantage over dual flaps, as opposed to the results shown previously using $2-5 /$ rev only. The flap deflections are shown in Fig. 18, and their respective harmonic compositions are displayed in Fig. 19. In the single flap cases, the constant and $1 /$ rev components required by the controller are relatively small. In fact, in the single flap case with constant component, as shown in Fig. 19a, the required 0/rev component is negligible. Comparing the degrees of noise reduction by including the $0 / \mathrm{rev}$ or $1 / \mathrm{rev}$ components with those that can be obtained using the traditional $2-5 / \mathrm{rev}$ only, it is evident that the additional $0 / \mathrm{rev}$ or $1 / \mathrm{rev}$ components do not have significant effects on BVI noise reduction, with the current active flap configurations.

\section{Results for MD-900 Bearingless Rotor}

In this section the simulation results are presented, using the rotor and flap configuration of the modified MD-900 rotor developed in the SMART program. The purpose of these simulations is to examine a different type of rotor configuration and thus display the simulation capabilities of the code. This goal is also expanded by considering a number of parameters that would influence the practical implementation of ACF system on an actual bearingless rotor. The parabolic tapering of the blade tip in the actual rotor, which is designed for noise and vibration benefits during high speed flight, is not considered in the current simulation for the low speed flight regime. The baseline case represents descending flight condition at $\mu=0.2$ and a thrust coefficient of $C_{T} / \sigma=0.0774$. The rotor is trimmed to zero first harmonic blade flapping and the specified $C_{T} / \sigma$ with a $-3.5^{\circ}$ aft tip path plane angle, using wind tunnel trim option. Note that for this study a plain flap configuration, as developed in the SMART program, is used as opposed to the servo flap used in the previous cases for the MBB BO-105 rotor. The properties of the rotor and flap configurations are summarized below in Tables 4 and 5. These can also be found in Ref. 24, and some additional data were provided by Dr. Friedrich Straub from Boeing Company, Mesa, AZ.

\section{A. Effects of Flap Overhang}

The effects of aerodynamic balance (flap overhang) are investigated in this section. Flap overhang is defined as the offset between the flap hinge and the leading edge of the flap, typically given as a percentage of 

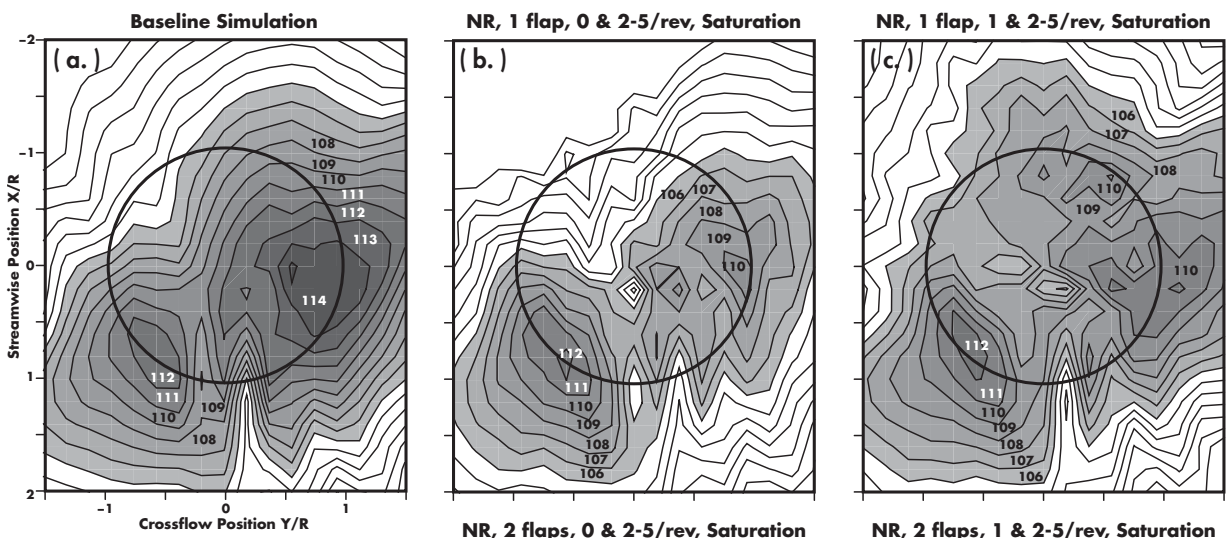

NR, 2 flaps, 0 \& 2-5/rev, Saturation
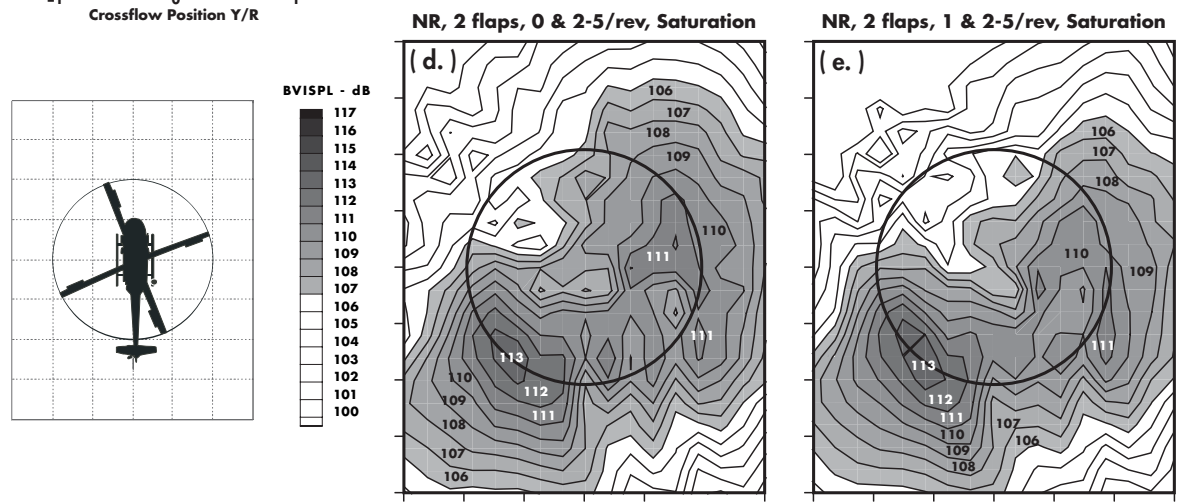

Figure 17. Carpet plot for noise reduction with $4^{\circ}$ saturation limits, effects of constant and $1 /$ rev pitch inputs.
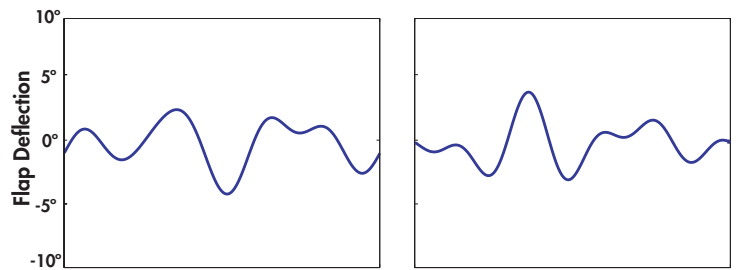

(a) Single flap, 0 \& 2-5/rev

(b) Single flap, $1 \& 2-5 /$ rev
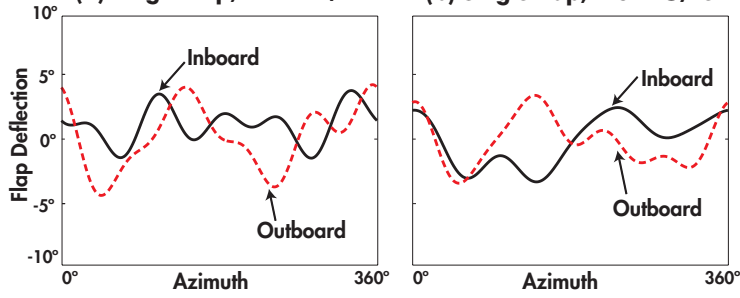

(c) Dual flaps, 0 \& 2-5/rev

(d) Dual flaps, $1 \& 2-5 /$ rev

Figure 18. Flap deflections for noise reduction, the effects of constant and $1 /$ rev pitch inputs. 


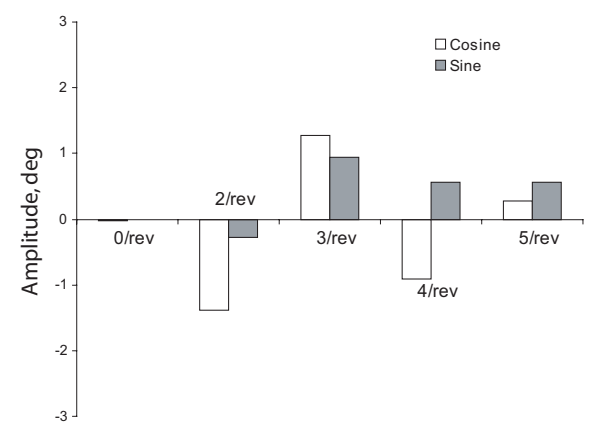

(a) NR, 1 flap, 0 \& 2-5/rev

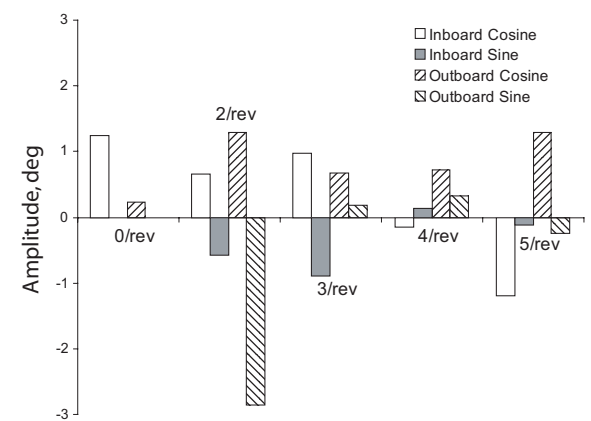

(c) NR, 2 flaps, 0 \& 2-5/rev

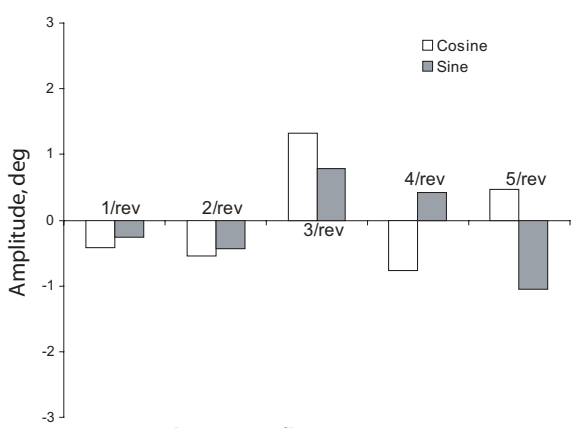

(b) NR, 1 flap, 1 \& 2-5/rev

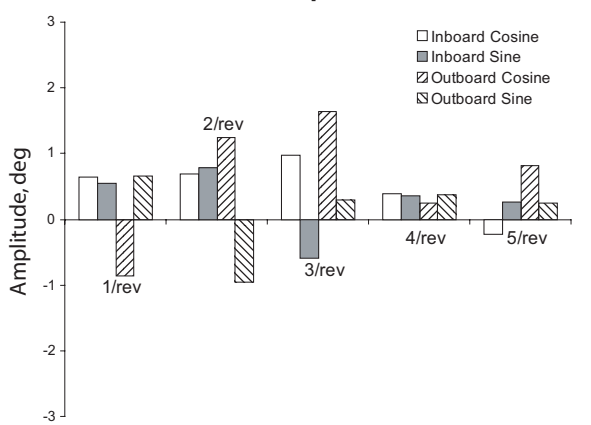

(d) NR, 2 flaps, $1 \& 2-5 /$ rev

Figure 19. Flap deflection harmonic compositions for noise reduction.

Table 4. MD-900 bearingless rotor configuration.

\begin{tabular}{ll}
\hline$\underline{\text { Rotor Data }}$ & \\
$N_{b}=5$ & $c=0.04924 L_{b}$ \\
$\omega_{F}=1.04,2.61,4.50$ & $C_{d o}=0.01$ \\
$\omega_{L}=0.65,3.51$ & $C_{m o}=0.0$ \\
$\omega_{T}=5.67$ & $a_{o}=2 \pi$ \\
$\theta_{\mathrm{tw}}=-10^{\circ}$ & $\alpha^{\prime}=-3.5^{\circ}$ \\
$\gamma=5.6$ & $\sigma=0.078$ \\
$\beta_{p}=3^{\circ}$ & \\
Helicopter Data & \\
\hline$C_{T} / \sigma=0.0774$ & $\mu=0.20$ \\
$L_{b}=5.16 \mathrm{~m}$ & $M_{\mathrm{ref}}=27.58 \mathrm{~kg}$ \\
$\Omega=392 \mathrm{RPM}$ & \\
\hline
\end{tabular}

Table 5. Flap configuration.

$c_{c}=0.25 c$

Single Plain Flap

$x_{c}=0.83 L_{b} \quad L_{c}=0.18 L_{b}$ 
the flap chord. It has been shown that flap overhang is an important design parameter for the practical implementation of the active flap system, which can significantly reduce the flap hinge moment and thus actuation power. ${ }^{20,24}$ In this section, the flap motion is prescribed at a single frequency of $4 /$ rev with maximum $2^{\circ}$ amplitude, $\delta_{f}=2 \cos (4 \psi-240)$.

The calculated flap hinge moments without overhang and with $40 \%$ overhang are compared in Fig. 20. Clearly the $40 \%$ overhang significantly reduces the required hinge moment. The current simulation captured a similar trend but underpredicted the hinge moment, when compared to the result obtained in Ref. 20 using CAMRAD II with table lookup aerodynamics. Another theoretical study of active flap with overhang using UMARC $^{42}$ also indicated that hinge moment is underpredicted, suggesting that it is difficult to theoretically model the flap hinge moment.

The instantaneous power required to drive the flap over one rotor revolution is shown in Fig. 21 . It is evident that with $40 \%$ overhang the power requirement needed for actuation is reduced by nearly an order of magnitude.

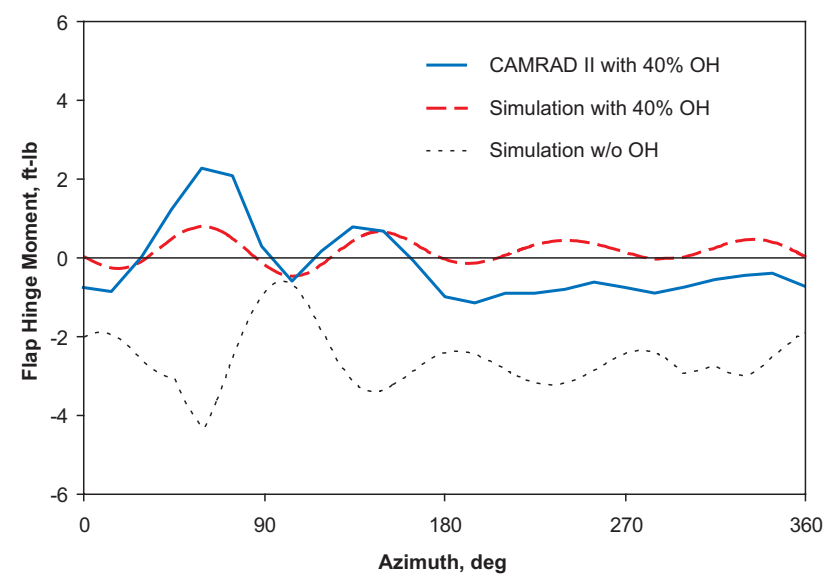

Figure 20. Effect of flap overhang on hinge moment, with comparison to CAMRAD II.

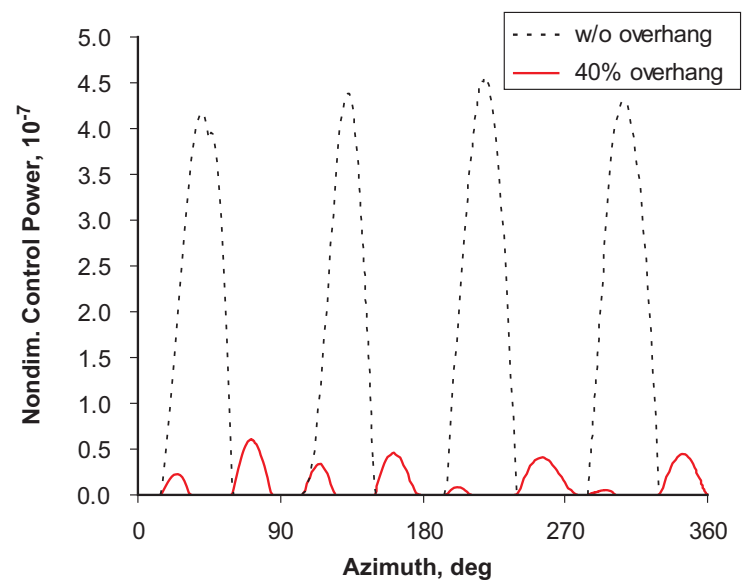

Figure 21. Instantaneous control power requirement over one revolution, with and without flap overhang.

\section{B. Vibration Reduction}

First the active flap is considered exclusively for vibration reduction. The control objective consists of $5 / \mathrm{rev}$ vibratory hub loads since the main rotor is five-bladed and $5 / \mathrm{rev}$ vibratory components are dominant. The flap deflection harmonic components of interest for active control, in this case, are $2 / \mathrm{rev}, 3 / \mathrm{rev}, 4 / \mathrm{rev}$, 
$5 / \mathrm{rev}, 6 / \mathrm{rev}$. Moreover, the maximum flap deflection is restricted to $4^{\circ}$ with saturation limits enforced. The characteristics of the rotor and input used for vibration reduction resemble some aspects of the active flap developed in the SMART program.

Figure 22 shows 5/rev vibratory hub loads, for the baseline uncontrolled case and together with the actively controlled values after vibration reduction. The active flap produces vibration reduction of nearly $60 \%$ in the overall vibration objectives, under the simulated descending flight condition where the BVI interaction is strong. This demonstrates the effectiveness of the ACF on the five-bladed bearingless rotor despite the substantial differences when compared to the four-bladed hingeless MBB BO-105 rotor discussed previously.

The effect of vibration reduction on noise is considered next. The noise carpet plots on a plane located $1.15 R$ below the hub plane are shown in Fig. 23 for the baseline and vibration controlled cases. Similar to the results for the MBB BO-105, the peak advancing side noise during vibration reduction is slightly increased by about $1 \mathrm{~dB}$.

The flap deflection time history is shown in Fig. 24, with the maximum deflection limited to $4^{\circ}$. The flap deflection differs significantly from those shown in Fig. 10 for the MBB BO-105 case, however, it comes at no surprise considering the different size, location of the active flap and the control objective ( $5 /$ rev vs. $4 /$ rev hub loads).

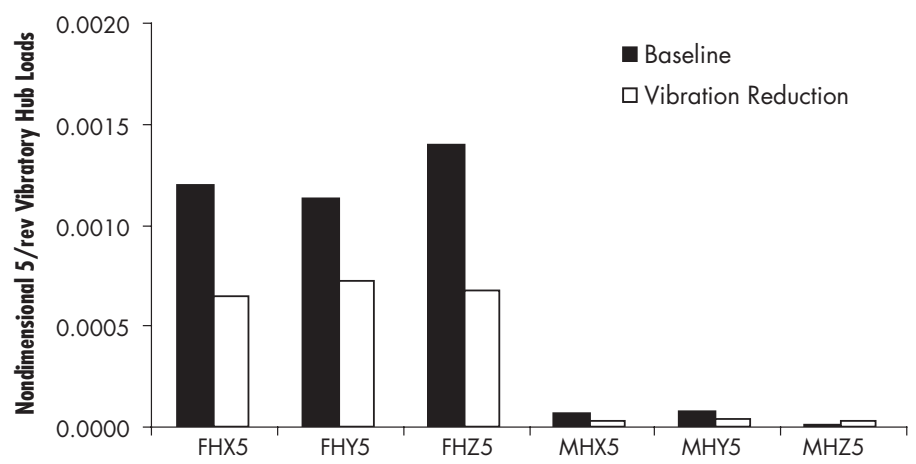

Figure 22. The $5 /$ rev vibratory loads, the baseline and with vibration reduction.
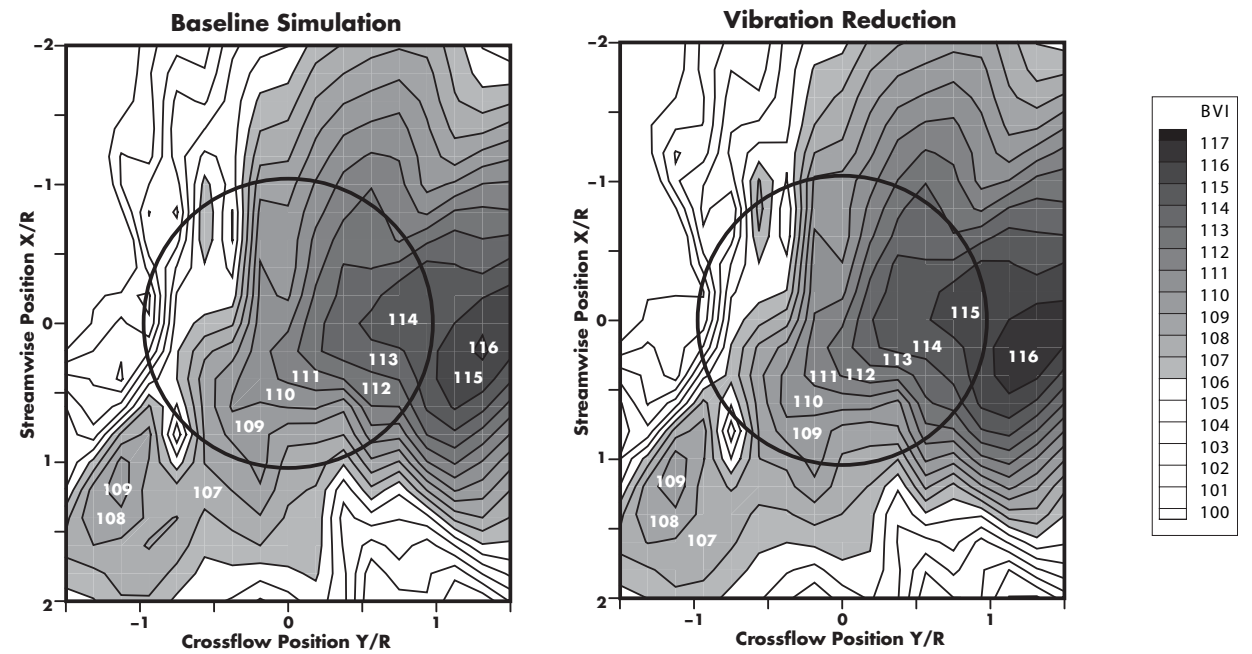

Figure 23. Noise carpet plots, the baseline and with vibration reduction. 


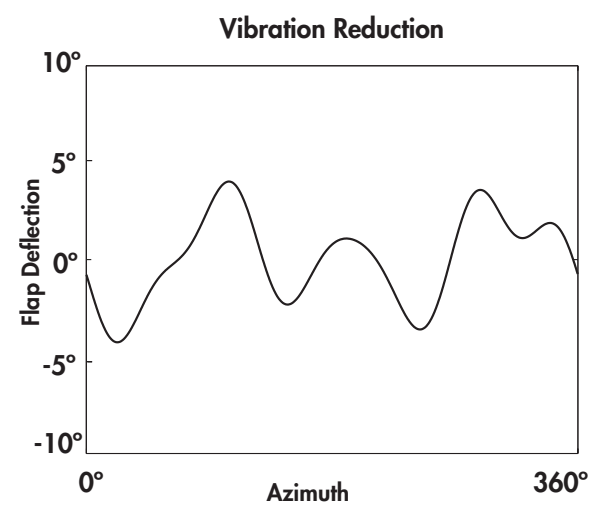

Figure 24. Flap deflection during vibration reduction.

\section{Noise Reduction}

Next, the adaptive control algorithm, with $4^{\circ}$ saturation limits, is used to reduce the noise levels at the SKID-REAR microphone location, using flap harmonic components between 2-6/rev. The peak advancing side noise at the carpet plane is reduced by $3 \mathrm{~dB}$, as can been in Fig. 25. Meanwhile the retreating side noise is not increased as opposed to the behavior of the MBB BO-105 hingeless rotor case. Instead, the BVI noise on the whole carpet plane is reduced uniformly. This demonstrates the effectiveness of ACF for noise reduction on the bearingless rotor configuration.

The vibratory loads during noise reduction are depicted in Fig. 26. It can been seen that the noise reduction is associated with significant vibration penalty. All vibratory components are increased and the overall vibration objective is increased by $150 \%$. This is similar to the noise control case for the BO-105 using dual flap control. The required flap deflection is shown in Fig. 27.
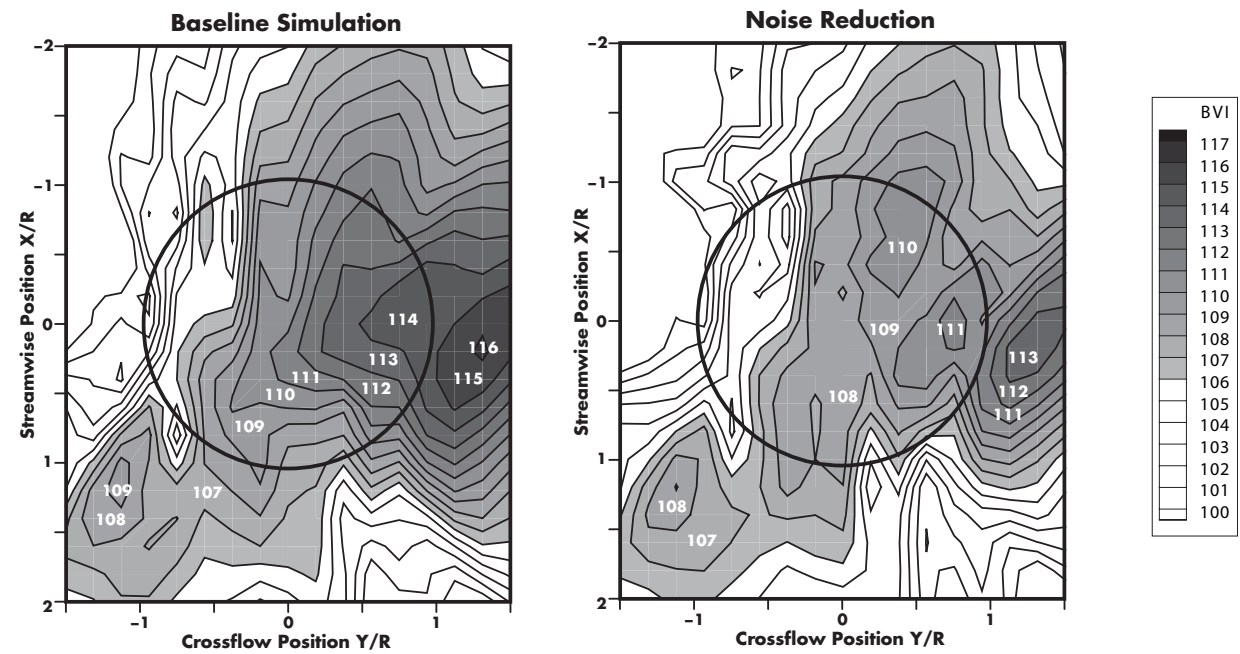

Figure 25. Noise carpet plots, the baseline and with noise reduction.

\section{Simultaneous Reduction}

Finally, simultaneous vibration and noise reduction using the active flap is considered. Again, the flap harmonic components are 2-6/rev. The vibratory loads and noise carpet plots after applying simultaneous control are shown in Figs. 28 and 29, respectively. Although the longitudinal and lateral hub shears increase slightly, the vertical vibration component is reduced by $74 \%$ and the resulting vibration objective function is 


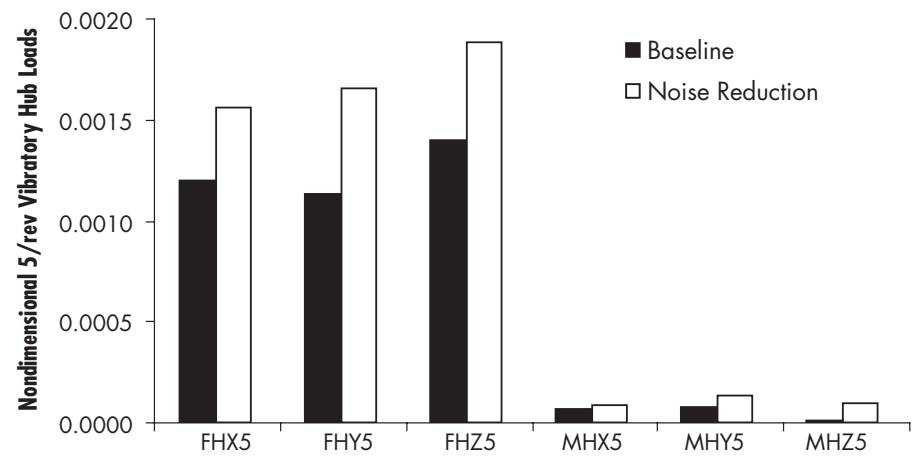

Figure 26. The 5/rev vibratory loads, the baseline and active noise control case.

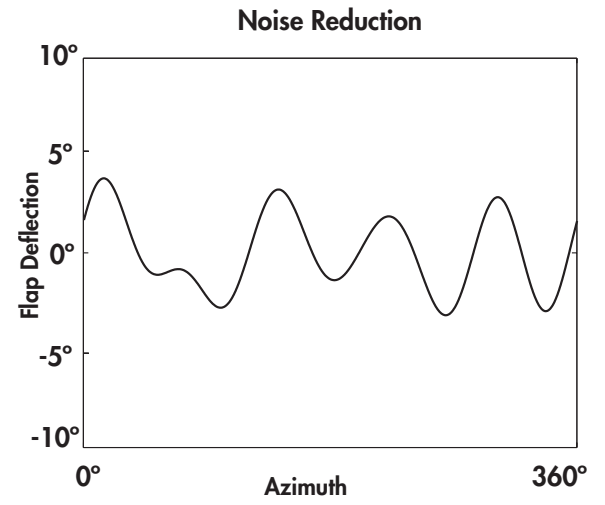

Figure 27. Flap deflection during noise reduction. 
reduced by about $8 \%$. Meanwhile, BVI noise is reduced by $1-2 \mathrm{~dB}$. Overall, the combined vibration and noise objective is reduced by approximately $30 \%$, representing a compromise solution. Comparing these with the simultaneous noise and vibration reduction observed on a hingeless rotor similar to the MBB BO- $105,{ }^{26,27}$ it appears that the noise reductions obtained for the bearingless rotor are smaller. However, this may also be due to the use of the plain flap, for the bearingless rotor case, which is known to be less effective than the servo flap based ACF system used for the other cases studied.

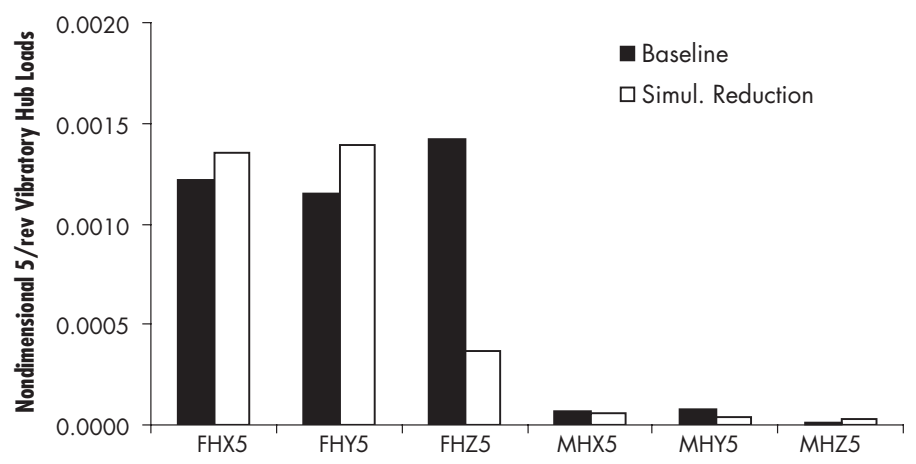

Figure 28. The 5/rev vibratory loads, the baseline and with simultaneous reduction.
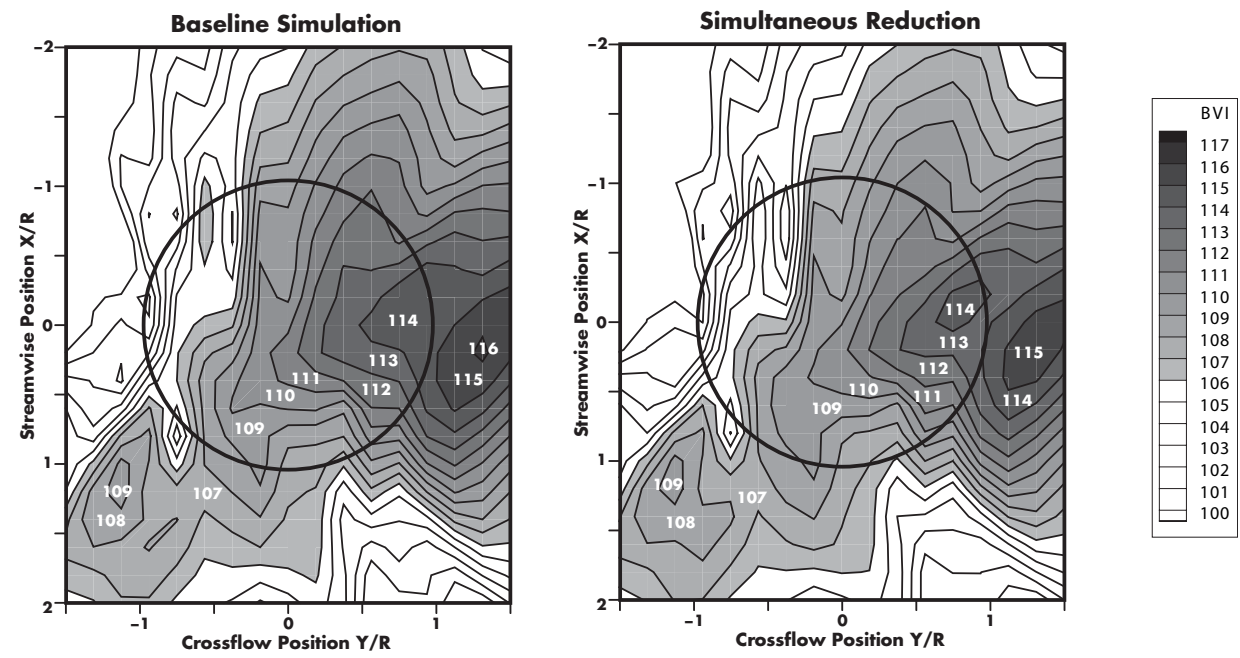

Figure 29. Noise carpet plots, the baseline and with simulteneous reduction.

\section{E. Effect of Swept Tips}

Tip sweep and anhedral have been shown in previous studies ${ }^{17}$ to have beneficial effects on the vibratory vertical hub shears, for a hingeless rotor resembling MBB BO-105. The effects of advanced tip shapes including tip sweep and anhedral on the vibration and BVI noise are considered in this section, when using the MD-900 bearingless rotor. The swept tip is modeled using a single finite element, with $10 \%$ of the blade length. Tip sweep angle $\Lambda_{s}$ is defined positive backward, and tip inclination angle from the horizontal $\Lambda_{a}$ is defined positive upward.

The effect of the swept tips on the $5 /$ rev vibratory loads is shown in Fig. 31, with the rotor trimmed to $-3.5^{\circ}$ aft tip path plane angle and an advance ratio of 0.2 . This is identical to the baseline case in the active control results; simulating descending flight condition. Three tip configurations are examined: $\Lambda_{s}=10^{\circ}$, $\Lambda_{a}=10^{\circ}$ (dihedral) and $\Lambda_{a}=-10^{\circ}$ (anhedral). It is clear that dihedral and anhedral have a significant effect on the vibratory loads. The $10^{\circ}$ anhedral affects the vibratory loads adversely, with $34 \%$ vibration increase 


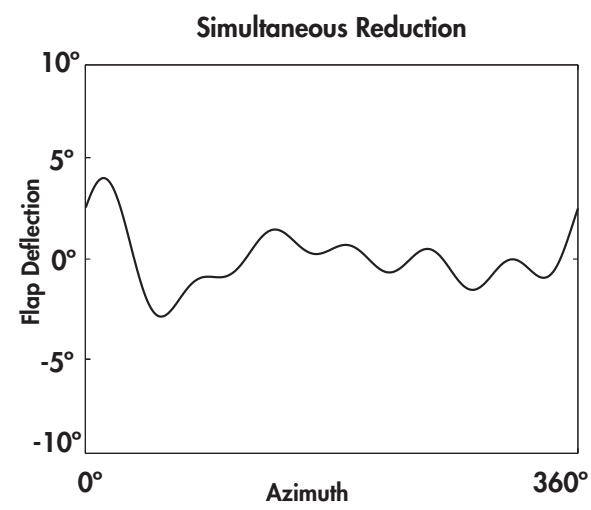

Figure 30. Flap deflection during simulteneous reduction.

in the vertical hub shear. Meanwhile, the vertical shear is reduced by $40 \%$ using the $10^{\circ}$ dihedral. The results differ from the previous findings that the anhedral is beneficial for vibration levels. This is due to the simulated descending flight condition where the rotor tip path plane tilts backward and the blade-vortex separation distance is increased by the dihedral, whereas the separation distance is increased by the anhedral when the rotor tip path plane tilts forward; such as in level flight condition. The increased blade-vortex separation distance is beneficial for BVI alleviation while the reduced blade-vortex separation distance is detrimental. To demonstrate this, the effects of tip sweep and anhedral for the same rotor, trimmed to $2^{\circ}$ tip path plane angle, simulating level flight condition are presented in Fig. 32. It is clear that in level flight condition the anhedral has beneficial effects while the dihedral is detrimental. This agrees with the results given in Ref. 17, obtained under the level flight condition. The tip sweep has a small effect on the vibratory loads, for the low advance ratio flight condition considered, as can be seen in Figs. 31 and 32.

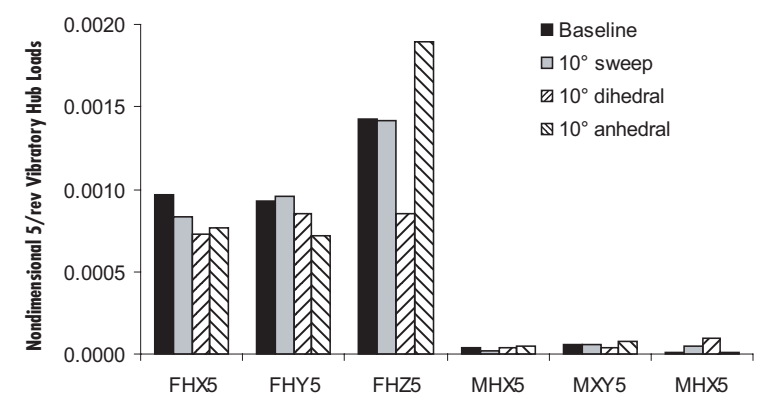

Figure 31. The effects of tip sweep, dihedral and anhedral on vibration, $\alpha^{\prime}=-3.5^{\circ}, \mu=0.2$, simulated descent flight condition.

The BVI noise contours are presented in Fig. 33 for the simulated descending flight and Fig. 34 for the simulated level flight. It can be seen in Fig. 33 that there are small BVI noise benefits of up to $1 \mathrm{~dB}$ for the tip sweep and the $10^{\circ}$ dihedral cases, while the $10^{\circ}$ anhedral produces slight increase in noise, under the descending flight condition. By comparison, for level flight the $10^{\circ}$ dihedral produces $3 \mathrm{~dB}$ increase in BVI noise whereas the $10^{\circ}$ anhedral provides $2 \mathrm{~dB}$ reduction in noise, as can be seen in Fig. 34. It is worth mentioning that the BVI noise levels are much smaller under the level flight condition when compared to the descending flight, where the BVI effects are most intensive. Comparing the effectiveness of the swept tips under heavy BVI conditions to the results shown previously when using the ACF, it is evident that the active control approach provides superior noise reduction. It should be noted that swept tip is expected to have more appreciable benefits during high speed flight, although its effects on the BVI noise are small for low advance ratio flight regime considered here. 


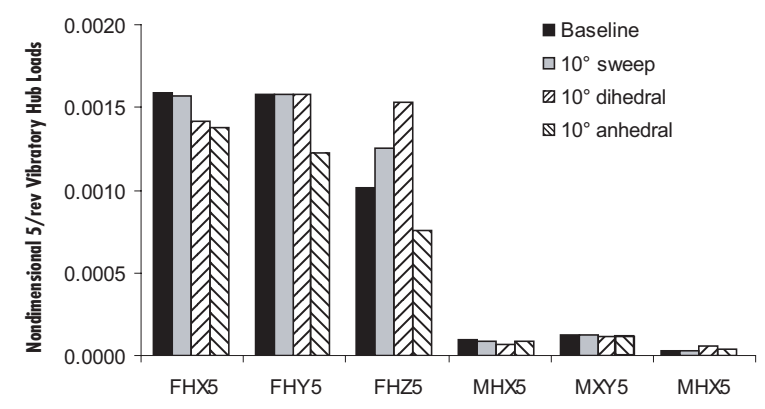

Figure 32. The effects of tip sweep, dihedral and anhedral on vibration, $\alpha^{\prime}=2^{\circ}, \mu=0.2$, simulated level flight condition.

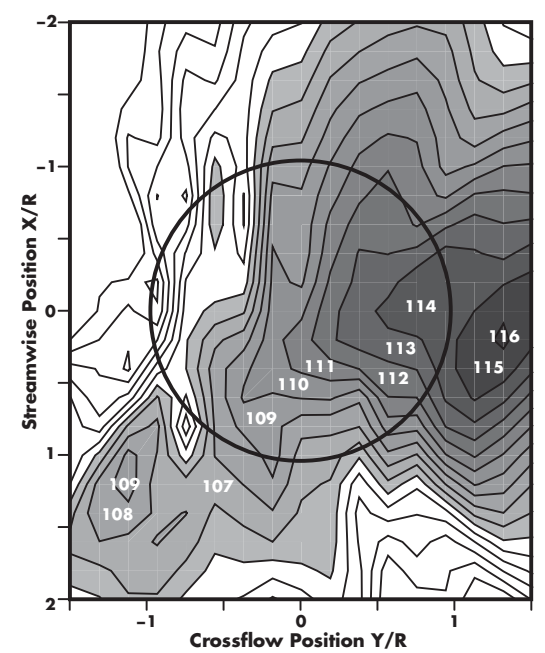

(a). Baseline Simulation

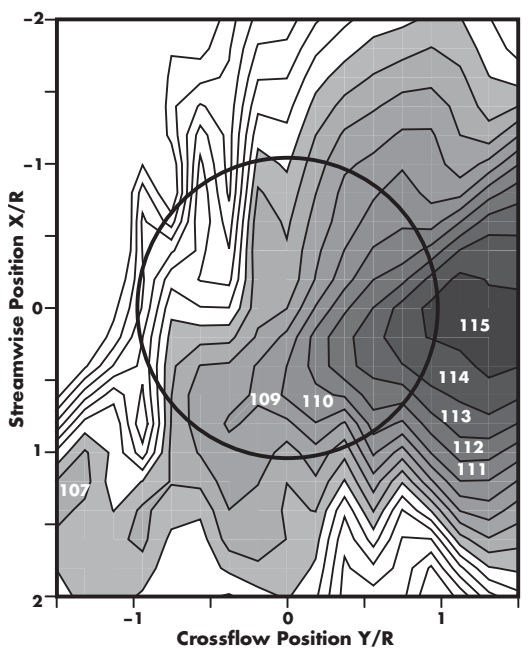

(c). $10^{\circ}$ Dihedral

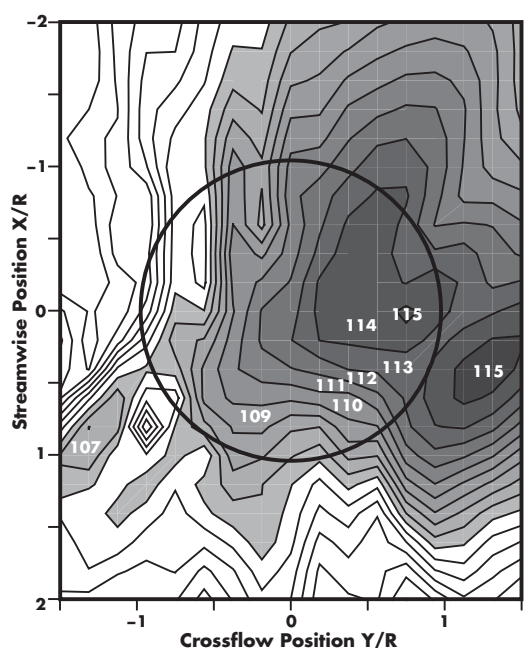

(b). $10^{\circ}$ Sweep

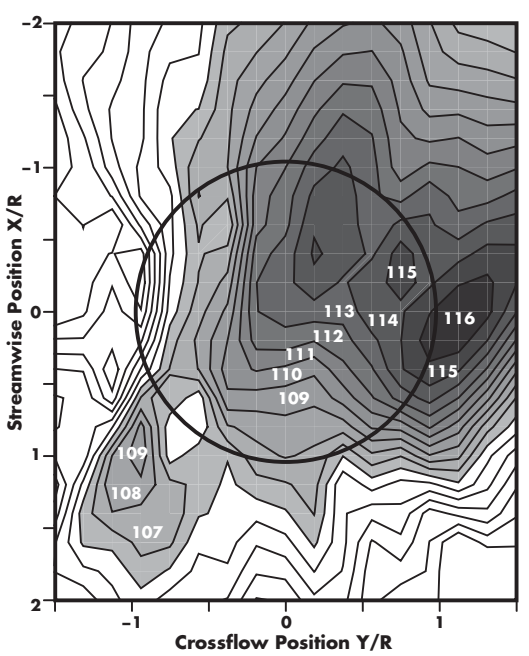

(d). $10^{\circ}$ Anhedral

Figure 33. The effects of tip sweep, dihedral and anhedral on noise, $\alpha^{\prime}=-3.5^{\circ}, \mu=0.2$, simulated descent flight condition. 


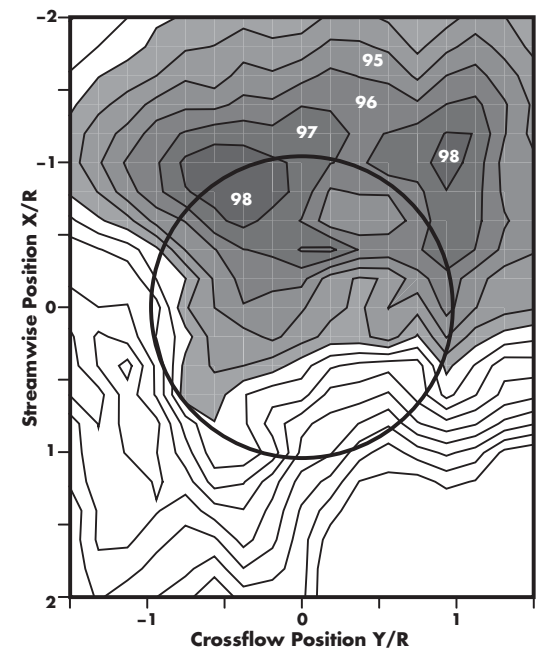

(a). Baseline Simulation

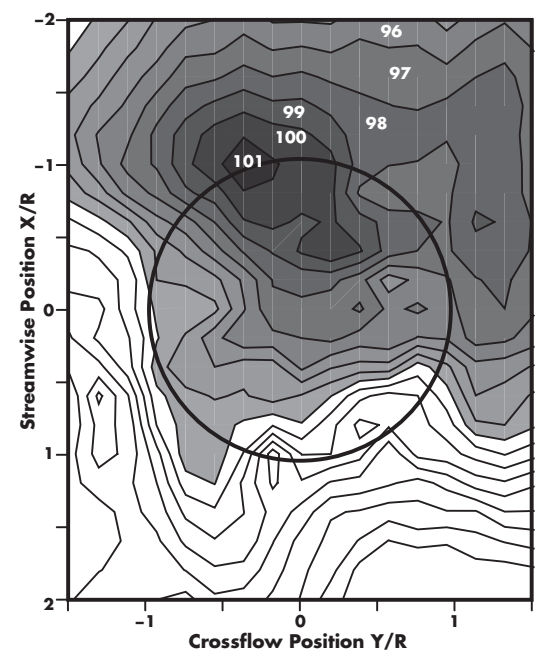

(c). $10^{\circ}$ Dihedral

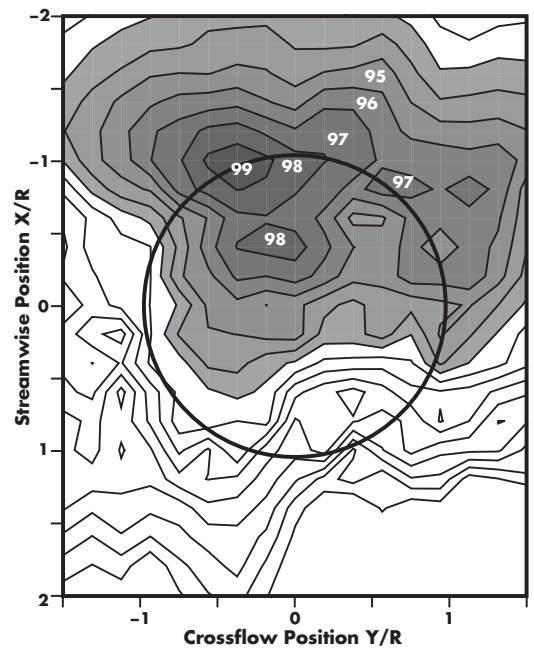

(b). $10^{\circ}$ Sweep

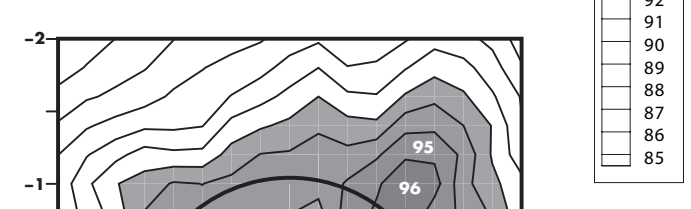

Figure 34. The effects of tip sweep, dihedral and anhedral on noise, $\alpha^{\prime}=2^{\circ}, \mu=0.2$, simulated level flight condition. 


\section{Conclusions}

The results presented in this paper have demonstrated that the ACF system implemented in a single or dual flap configuration is an effective means of reducing vibration and BVI noise. The numerical simulations conducted have also indicated that the ACF system is effective on two substantially different rotor configurations. The key results for two rotors with a number of flap configurations are summarized in Table 6 .

Table 6. Summary of key results from two rotor configurations, with $4^{\circ}$ saturation limits.

\begin{tabular}{l|cccccc|ccc} 
Rotor & \multicolumn{5}{|c|}{ BO-105 } & \multicolumn{3}{c}{ MD-900 } \\
\hline Type & \multicolumn{4}{c}{ Hingeless } & \multicolumn{4}{c}{5} \\
$N_{b}$ & \multicolumn{4}{c}{ single plain } \\
Flap No. \& Type & \multicolumn{3}{|c}{ single servo } & \multicolumn{3}{c}{ dual servo } & \\
Controller Objective & VR & NR & SR & VR & NR & SR & VR & NR & SR \\
$\Delta$ Vibration $^{\dagger}$ & $-46 \%$ & $+2 \%$ & $-57 \%$ & $-86 \%$ & $+130 \%$ & $-44 \%$ & $-60 \%$ & $+150 \%$ & $-8 \%$ \\
$\Delta$ BVI Noise $^{\ddagger}$ & $-1 \mathrm{~dB}$ & $-6 \mathrm{~dB}$ & $-3 \mathrm{~dB}$ & $-1 \mathrm{~dB}$ & $-5 \mathrm{~dB}$ & $-4 \mathrm{~dB}$ & $+1 \mathrm{~dB}$ & $-4 \mathrm{~dB}$ & $-2 \mathrm{~dB}$ \\
\hline
\end{tabular}

$\dagger$ Changes in vibration objective.

${ }^{\ddagger}$ BVI noise changes at SKID-REAR location.

The primary conclusions that can be drawn from this study are summarized below:

1. The ACF is an effective device for vibration and BVI noise reduction in rotorcraft, for different types of rotors and different helicopter configurations.

2. The effectiveness of the ACF system has been clearly demonstrated despite imposing a practical saturation limit of $4^{\circ}$ on the maximum flap deflections allowed.

3. The addition of constant and $1 /$ rev flap harmonic input to the harmonic content of flap deflection used does not have significant effects on BVI noise reduction, for the active flap systems employed on a rotor that resembles the MBB BO-105 rotor.

4. Using a substantial flap overhang is a very effective means of reducing the flap hinge moments, thus further reducing the actuation power requirement for the ACF system; which is already low when compared to HHC or traditional IBC.

5. A passive approach is also explored in this paper by varying the sweep and anhedral on the tip of the rotor, a common practice in advanced geometry rotors. The anhedral(dihedral) is more effective at alleviating the BVI effects than the tip sweep, and its effectiveness is due to increased blade vortex separation distance. However, this reduction depends on the flight conditions. A $10^{\circ}$ dihedral can provide $40 \%$ reduction in vertical hub shear under descending flight conditions with a negative tip path plane angle, while it is detrimental in the level flight with a positive tip path plane angle. The $10^{\circ}$ anhedral provides $25 \%$ reduction in vertical shear and $2 \mathrm{~dB}$ noise reduction under the level flight conditions while it is detrimental in the descending flight.

6. The ACF provides superior vibration and BVI noise reduction compared to the passive approach.

\section{Acknowledgments}

This research was supported by the FXB Center for Rotary and Fixed Wing Air Vehicle Design, and by ARO grant 02-1-0202 with Dr. G. Anderson as grant monitor.

\section{References}

\footnotetext{
${ }^{1}$ Friedmann, P. P. and Millott, T. A., "Vibration Reduction in Rotorcraft Using Active Control: A Comparison of Various Approaches," Journal of Guidance, Control, and Dynamics, Vol. 18, No. 4, July-August 1995, pp. 664-673.
} 
${ }^{2}$ Friedmann, P. P., "Rotary Wing Aeroelasticity - Current Status and Future Trends," AIAA Journal, Vol. 42, No. 10, Oct. 2004, pp. 1953-1972.

${ }^{3}$ Yu, Y. H., "Rotor Blade-Vortex Interaction Noise," Progress in Aerospace Sciences, Vol. 36, No. 2, Feb. 2000 , pp. $97-115$.

${ }^{4}$ Brooks, T. F., Booth, E. R., Jolly, J. R., Yeager, W., and Wilbur, M., "Reduction of Blade-Vortex Interaction Noise Through Higher Harmonic Pitch Control," Journal of the American Helicopter Society, Vol. 35, No. 1, Jan. 1990 , pp. 86-91.

${ }^{5}$ Brooks, T. F. and Booth, E. R., "The Effect of Higher Harmonic Pitch Control on Blade-Vortex Interaction Noise and Vibration," Journal of the American Helicopter Society, Vol. 38, No. 3, July 1993, pp. 45-55.

${ }^{6}$ Splettstoesser, W., Schultz, K. J., Kube, R., Brooks, T. F., Booth, E. R., Niesl, G., and Streby, O., "A Higher Harmonic Control Test in the DNW to Reduce Impulsive BVI Noise," Journal of the American Helicopter Society, Vol. 39, No. 4, Oct. 1994, pp. 3-13.

${ }^{7}$ Splettstoesser, W., Kube, R., Wagner, W., Seelhorst, U., Boutier, A., Micheli, F., Mercker, E., and Pengel, K., "Key Results From a Higher Harmonic Control Aeroacoustic Rotor Test (HART)," Journal of the American Helicopter Society, Vol. 42, No. 1, Jan. 1997, pp. 58-78.

${ }^{8}$ Yu, Y. H., Tung, C., van der Wall, B., Pausder, H. J., Burley, C., Brooks, T., Beaumier, P., Delrieux, Y., Mercker, E., and Pengel, K., "The HART-II Test: Rotor Wakes and Aeroacoustics with Higher-Harmonic Pitch Control (HHC) Inputs The Joint German/French/Dutch/US Project -," Proceedings of the 58th Annual Forum of the American Helicopter Society, Montreal, Canada, June 2002.

${ }^{9}$ Jacklin, S. A., "Second Test of a Helicopter Individual Blade Control System in the NASA Ames 40 by 80 Foot Wind Tunnel," Proceedings of the 2nd International American Helicopter Society Aeromechanics Specialists Conference, Bridgeport, CT, Oct. 1995.

${ }^{10}$ Swanson, S. M., Jacklin, S. A., Blaas, A., Niesl, G., and Kube, R., "Reduction of Helicopter BVI Noise, Vibration, and Power Consumption through Individual Blade Control," Proceedings of the 51st Annual Forum of the American Helicopter Society, Fort Worth, TX, May 1995, pp. 662-680.

${ }^{11}$ Jacklin, S. A., Blaas, A., Teves, D., and Kube, R., "Acoustic Results from a Full-Scale Wind Tunnel Test Evaluating Individual Blade Control," Proceedings of the 51st Annual Forum of the American Helicopter Society, Fort Worth, TX, May 1995, pp. 461-480.

12 Jacklin, S. A., Haber, A., de Simone, G., Norman, T., Kitaplioglu, C., and Shinoda, P., "Full-Scale Wind Tunnel Test of an Individual Blade Control System for a UH-60 Helicopter," Proceedings of the 51st Annual Forum of the American Helicopter Society, Montreal, Canada, June 2002.

${ }^{13}$ Bebesel, M., Roth, D., Dietrich, R., Pongratz, K., and Kube, R., "Individual Blade Root Control Demonstration Recent Activities," Proceedings of the 27th European Rotorcraft Forum, Moscow, Russia, Sept. 2001, pp. 33.1-33.14.

${ }^{14}$ Bebesel, M., Roth, D., and Pongratz, K., "Reduction of BVI Noise Ground - In Flight Evaluation of Closed-Loop Controller," Proceedings of the 28th European Rotorcraft Forum, Bristol, England, Sept. 2002, pp. 19.1-19.9.

${ }^{15}$ Millott, T. A. and Friedmann, P. P., Vibration Reduction in Helicopter Rotors Using an Actively Controlled Partial Span Trailing Edge Flap Located on the Blade, NASA CR 4611, June 1994.

${ }^{16}$ Myrtle, T. F. and Friedmann, P. P., "Application of a New Compressible Time Domain Aerodynamic Model to Vibration Reduction in Helicopters Using an Actively Controlled Flap," Journal of the American Helicopter Society, Vol. 46, No. 1, Jan. 2001, pp. 32-43.

${ }^{17}$ de Terlizzi, M. and Friedmann, P. P., "Active Control of BVI Induced Vibrations Using a Refined Aerodynamic Model and Experimental Correlation," Proceedings of the 51st Annual Forum of the American Helicopter Society, Fort Worth, TX, May 1995, pp. 510-524.

${ }^{18}$ Depailler, G. and Friedmann, P. P., "Reductions of Vibrations Due to Dynamic Stall in Helicopters Using an Actively Controlled Flap," Proceedings of the 43rd AIAA/ASME/ASCE/AHS/ACS Structures, Structural Dynamics and Materials Conference, Denver, CO, April 2002, AIAA Paper No. 2002-1431.

${ }^{19}$ Friedmann, P. P., de Terlizzi, M., and Myrtle, T. F., "New Developments in Vibration Reduction with Actively Controlled Trailing Edge Flaps," Mathematical and Computer Modelling, Vol. 33, 2001, pp. 1055-1083.

${ }^{20}$ Straub, F. K. and Charles, B. D., "Aeroelastic Analysis of Rotors with Trailing Edge Flaps Using Comprehensive Codes," Journal of the American Helicopter Society, Vol. 46, No. 3, July 2001, pp. 192-199.

${ }^{21}$ Koratkar, N. A. and Chopra, I., "Wind Tunnel Testing of a Smart Rotor Model with Trailing Edge Flaps," Journal of the American Helicopter Society, Vol. 47, No. 4, Oct. 2002, pp. 263-272.

${ }^{22}$ Fulton, M. and Ormiston, R. A., "Small-Scale Rotor Experiments with On-Blade Elevons to Reduce Blade Vibratory Loads in Forward Flight," Proceedings of the 54th Annual Forum of the American Helicopter Society, Washington, DC, May 1998, pp. 433-451.

${ }^{23}$ Straub, F. K., Kennedy, D. K., Bomzalski, D. B., Hassan, A. A., Ngo, H., Anand, V. R., and Birchette, T. S., "Smart Material Actuated Rotor Technology - SMART," Proceedings of the 41th Structures, Structural Dynamics and Materials Conference, Atlanta, GA, April 2000, AIAA Paper No. 2000-1715.

${ }^{24}$ Straub, F. K., Kennedy, D. K., Stemple, A. D., Anand, V. R., and Birchette, T. S., "Development and Whirl Tower Test of the SMART Active Flap Rotor," Proceedings of SPIE: Smart Structures and Materials 2004, Vol. 5388, July 2004, pp. 202-212.

${ }^{25}$ Patt, D., Liu, L., and Friedmann, P. P., "Rotorcraft Vibration Reduction and Noise Prediction Using a Unified Aeroelastic Response Simulation," Journal of the American Helicopter Society, Jan. 2005.

${ }^{26}$ Liu, L., Patt, D., and Friedmann, P. P., "Simultaneous Vibration and Noise Reduction in Rotorcraft Using Aeroelastic Simulation," Proceedings of the 60th American Helicopter Society Annual Forum, Baltimore, MD, June 2004.

${ }^{27}$ Patt, D., Liu, L., and Friedmann, P. P., "Achieving Simultaneous Noise and Vibration Reduction Using Simulation," Proceedings of the 30th European Rotorcraft Forum, Marseille, France, September 2004.

${ }^{28}$ Yuan, K. A. and Friedmann, P. P., Aeroelastic and Structural Optimization of Composite Helicopter Rotor Blades with Swept Tips, NASA CR 4665, June 1995. 
${ }^{29}$ USAAMCOM/AFDD TR 02-A-006, RCAS User's Manual, Version 2.0, June 2002.

${ }^{30}$ Johnson, W., CAMRAD/JA - A Comprehensive Analytical Model of Rotorcraft Aerodynamics and Dynamics, Vol I. Theory Manual, Johnson Aeronautics, Palo Alto, CA, 1988.

${ }^{31}$ Johnson, W., CAMRAD/JA - A Comprehensive Analytical Model of Rotorcraft Aerodynamics and Dynamics, Vol II. Users' Manual, Johnson Aeronautics, Palo Alto, CA, 1988.

${ }^{32}$ Depailler, G., Alleviation of Dynamic Stall Induced Vibrations on Helicopter Rotors Using Actively Controlled Flaps, Ph.D. thesis, University of Michigan, Ann Arbor, 2002.

${ }^{33}$ Brenter, K., A Computer Program Incorporating Realistic Blade Motions and Advanced Acoustic Formulation, NASA Technical Memorandum, Vol. 877211986.

${ }^{34}$ Patt, D., Liu, L., Chandrasekar, J., Bernstein, D. S., and Friedmann, P. P., "The Higher Harmonic Algorithm for Helicopter Vibration Reduction Revisited," Proceedings of the 45th AIAA/ASME/ASCE/AHS/ACS Structures, Structural Dynamics and Materials Conference, Palm Springs, CA, April 2004, AIAA Paper No. 2004-1948. Accepted for publication in the Journal of Guidance, Control and Dynamics, 2005.

${ }^{35}$ Johnson, W., Self-Tuning Regulators for Multicyclic Control of Helicopter Vibrations, NASA Technical Paper $1996,1982$.

${ }^{36}$ Kube, R., van der Wall, B., Schultz, and Splettstoesser, W., "IBC Effects on BVI Noise and Vibrations a Combined Numerical and Experimental Investigation," Proceedings of the 55th Annual Forum of the American Helicopter Society, Montreal, Canada, May 1999.

${ }^{37}$ Liu, L., Patt, D., and Friedmann, P. P., "Active Vibration and Noise Reduction in Rotorcraft Using an Aeroelastic Simulation," Proceedings of the American Helicopter Society 4 th Decennial Specialists Conference on Aeromechanics, San Francisco, CA, Jan 2004.

${ }^{38}$ Cribbs, R. and Friedmann, P. P., "Actuator Saturation and Its influence on Vibration Reduction by Actively Controlled Flaps," Proceedings of the 42nd AIAA/ASME/ASCE/AHS/ACS Structures, Structural Dynamics and Materials Conference, Seattle, WA, April 2001, AIAA Paper No. 2001-1467.

${ }^{39}$ Johnson, W., "Rotorcraft Dynamics Models for a Comprehensive Analysis," Proceedings of the 54 th Annual Forum of the American Helicopter Society, Washington, D.C., May 1998.

${ }^{40}$ Schmitz, F. H. and Sim, B. W., "Blade-Vortex Interaction (BVI) Noise: Retreating Side Characteristics, Sensitivity to Chordwise Loading and Unsteady Aerodynamics," Proceedings of the AHS Aeromechanics Specialists Meeting, Atlanta, GA, Nov. 2000.

${ }^{41}$ Wachspress, D. A. and Quackenbush, T. R., "BVI Noise Mitigation Via Steady Flap Deflection - An Analysis-Led Test Program," Proceedings of the American Helicopter Society 4 th Decennial Specialists Conference on Aeromechanics, San Francisco, CA, Jan 2004.

${ }^{42}$ Shen, J. and Chopra, I., "Aeroelastic Stability of Trailing-Edge Flap Helicopter Rotors," Journal of the American Helicopter Society, Vol. 48, No. 4, Oct. 2003, pp. 236-243. 